\title{
Intrinsic Limits of Dimensionality and Richness in Random Multipath Fields
}

\author{
Rodney A. Kennedy, Fellow, IEEE, Parastoo Sadeghi, Member, IEEE, Thushara D. Abhayapala, Member, IEEE, \\ and Haley M. Jones, Member, IEEE
}

\begin{abstract}
We study the dimensions or degrees of freedom of farfield multipath that is observed in a limited, source-free region of space. The multipath fields are studied as solutions to the wave equation in an infinite-dimensional vector space. We prove two universal upper bounds on the truncation error of fixed and random multipath fields. A direct consequence of the derived bounds is that both fixed and random multipath fields have an effective finite dimension. For circular and spherical spatial regions, we show that this finite dimension is proportional to the radius and area of the region, respectively. We use the Karhunen-Loève (KL) expansion of random multipath fields to quantify the notion of multipath richness. The multipath richness is defined as the number of significant eigenvalues in the KL expansion that achieve $99 \%$ of the total multipath energy. We establish a lower bound on the largest eigenvalue. This lower bound quantifies, to some extent, the well-known reduction of multipath richness with reducing the angular power spread of multipath angular power spectrum.
\end{abstract}

Index Terms-Multipath propagation, random scattering, spatial correlation function.

\section{INTRODUCTION}

\section{A. Motivation and Background}

W IRELESS communication systems use space as the physical medium for information transfer. The transmitted signal is often received via multiple paths due to reflection, diffraction, and scattering by objects in the wireless environment [1]. Using the spatial aspects of multipath is an increasingly active thread of research in wireless communications and signal processing [2]-[4]. This motivates studying the fundamental physical limits that space imposes on the dynamics of multipath wave propagation and wireless information transfer.

Manuscript received December 13, 2005; revised August 8, 2006. This work was supported by the Australian Research Council Discovery by Grant DP0343804. The material in this paper was presented in part at the IEEE International Conference on Acoustics, Speech, and Signal Processing, Orlando, FL, May 2002 and at the IEEE International Conference on Acoustics, Speech, and Signal Processing, Toulouse, France, May 2006. The associate editor coordinating the review of this manuscript and approving it for publication was Dr. Athanasios P. Liavas.

R. A. Kennedy and P. Sadeghi are with the Research School of Information Sciences and Engineering, The Australian National University, Canberra, ACT 0200 Australia (e-mail: rodney.kennedy@anu.edu.au; parastoo.sadeghi@anu. edu.au).

T. D. Abhayapala is with the Research School of Information Sciences and Engineering, The Australian National University, Canberra, ACT 0200 Australia. He is also with the National ICT Australia (e-mail: thushara. abhayapala@anu.edu.au).

H. M. Jones is with the Department of Engineering, Faculty of Engineering and Information Technology, The Australian National University, Canberra, ACT 0200 Australia (e-mail: haley.jones@anu.edu.au).

Digital Object Identifier 10.1109/TSP.2007.893738
The primary aim of this paper is to find the intrinsic limits on the dimensions or degrees of freedom for multipath fields when they are observed in, or coupled to a source-free region of space. This region of space is where multiple sensors may be potentially located to sample the multipath field for signal processing or communication purposes. However, our aim is to find universal bounds on multipath dimension without explicitly considering a specific sensor setup or application and hence, to show that the coupling of multipath into a spatial region is fundamentally limited by a finite number of orthogonal basis sets or modes. Throughout the paper, we will frequently refer to the radius or area of the two-dimensional (2-D) or threedimensional (3-D) multipath observation regions, respectively. In sensor array signal processing applications, an alternative terminology is the antenna aperture.

In this paper, we also aim to define spatial multipath richness. The predicted benefits of using spatial multipath in multiple-sensor wireless communications usually hinge on the imprecise assumption of rich multipath [5]-[7] or having a rich scattering environment. However, rich multipath needs to be mathematically quantified based on the dynamics of the multipath field random process and the wave equation. More specifically, we aim to precisely quantify the effects of multipath angular power spread and spatial observation region on richness, regardless of sensor setup.

Earlier works, [8], [9] introduced a general theoretical framework for studying the degrees of freedom in spatial multipath fields, where it was proposed that there is, essentially, a finite number of multipath that can be distinctively coupled to a source-free region. In another approach, [10] established a general analogy between the degrees of freedom in the time-frequency domain and in the spatial-angular domain for 2- and 3-D fields. It was concluded that the spatial-angular dimensionality is linearly related to the effective sensor array aperture and the angular power spread of scatterers.

\section{B. Approach}

The analysis in this paper considers multipath fields as the functional solutions to the wave equation [11]. This mathematical framework is similarly used in [8], [9], and [12]. The distinctive feature of this framework is that it directly considers the wavefield, which corresponds to continuous sampling in space, instead of a normal array model. In this presentation, the multipath field lies in a countable infinite-dimensional linear vector space, where vectors consist of functions. The advantage of the functional wave representation is that: 1) it is general enough to be applied to any narrowband multipath environment, regardless of the number or nature of multipath sources; 2) it accommodates representation of random multipath fields with a gen- 
eral spatial correlation function (SCF) [13]; and 3) it allows us to determine the effective number of dimensions (in the infinite-dimensional functional space) that essentially contribute to the coupling of multipath fields to a spatial region. In order not to obscure the approach, we present the main results for a narrowband 2-D multipath farfield environment. We will briefly explain how our methodology extends to 3-D fields. Unless explicitly stated, the equations and results in the paper are written assuming 2-D multipath fields.

The integral kernels for the spatial channel response in [10] and multipath field representations in this paper are both derived from the solutions to Maxwell equations. One difference is that [10] considers a zoomed-out granularity for describing channel scattering, where the total effect of scattering is modeled by the angular power spread in the kernels and appears in the expressions for scattering richness [10]. In this paper, however, scattering is directly incorporated into the multipath field representation. This formulation enables us to derive universal bounds on wavefield dimensionality or richness (Theorems 1 and 2) that hold equally well for any scattering angular power spread and with any angular power spectrum ${ }^{1}$ (APS), including isotropic scattering. Another difference is that [10] takes antenna polarization into account and shows that using tri-polarized arrays can result in a maximum twofold increase in multipath channel dimensionality. In this paper, we focus on unipolarized representation of multipath, where a similar conclusion as in [10] is expected by including polarization.

After deriving general bounds on scattering dimensionality, we wish to find bounds on scattering richness for a given multipath field with a known APS. It is known that the Karhunen-Loève (KL) expansion [14] of a random process allows a parsimonious representation/truncation of the process in the minimum mean square error (MMSE) sense. The KL expansion of a non-isotropic multipath field provides the maximally parsimonious and customized orthonormal expansion for that particular field. Therefore, we propose to use the KL expansion of random multipath fields to quantify their richness. In the KL expansion of multipath fields, the SCF eigenvalues and eigenfunctions play a central role. In particular, the number of significant SCF eigenvalues defines multipath richness, since random multipath is essentially generated by the corresponding significant eigenfunctions and an uncorrelated random sequence.

\section{Contributions}

The Dimension of Multipath Fields: In Section III, we establish two universal upper bounds on the truncation error of multipath fields in their infinite-dimensional presentation to a finite number of orthogonal modes. The first upper bound considers fixed multipath fields and complements the preliminary results in [8] by providing a mathematically rigorous proof. The second upper bound explicitly considers random multipath fields and upper bounds the field truncation mean square error (MSE). We show that for 2-D multipath fields, the truncation (mean square) error upper bound exponentially decays to zero, if the number of considered orthogonal modes is greater than a critical number

\footnotetext{
${ }^{1}$ The angular power spectrum quantifies the distribution of multipath power from different incident angles.
}

$2 N+1$. The critical value $N$ is directly related to the normalized radius of the 2-D region, $R / \lambda$ and is given as $N \triangleq\lceil\pi R e / \lambda\rceil$ ( $\lceil\cdot\rceil$ is the integer ceiling function and $\lambda$ is the wavelength). The results are noteworthy, as they show that the dimensionality in fixed or random 2-D multipath fields is essentially limited by the radius and not by the area of the 2-D observation/coupling region. The dimensionality in 3-D multipath fields is determined by $(N+1)^{2}$ and, hence, is related to the area and not to the volume of the 3-D region.

Multipath Richness: In Section IV, we use the multipath KL expansion to quantify the notion of multipath richness based on the number of significant SCF eigenvalues. More specifically, richness is defined as the number of SCF eigenvalues that capture at least $99 \%$ percent of the total multipath energy. This definition provides multipath richness results that are consistent with the $2\lceil\Omega R e / \lambda\rceil+1$ definition in [12], where $\Omega$ is the angular power spread. In [10], the channel dimensionality was shown to be $A|\Omega|$, for $R \gg 1$, where $A=2 R / \lambda$ is the normalized array aperture and $|\Omega|$ is the solid angular power spread of scatterers. We also establish a lower bound on the largest SCF eigenvalue. This lower bound quantifies, to some extent, the well-known qualitative effect of decreasing the angular power spread of scatterers on decreasing multipath richness.

\section{2-D Multipath Field RePRESENTATION}

Let $\boldsymbol{x}$ represent a vector in 2-D space, $\mathbb{R}^{2}$, and let $r=\|\boldsymbol{x}\|$ denote the Euclidean distance of $\boldsymbol{x}$ from the origin, which is the center of some region of interest. The unit vector in the direction of nonzero vector $\boldsymbol{x}$ is denoted $\widehat{\boldsymbol{x}} \triangleq \boldsymbol{x} /\|\boldsymbol{x}\|$. Further, let $\varphi(\boldsymbol{x}) \in$ $[0,2 \pi)$ represent the azimuth angle of vector $x$. Then, we can write $\widehat{\boldsymbol{x}}=[\cos \varphi(\boldsymbol{x}), \sin \varphi(\boldsymbol{x})]^{T}$, where $T$ denotes transpose. The vector $\boldsymbol{x}$ may also be represented in its polar form as

$$
\boldsymbol{x} \equiv(\|\boldsymbol{x}\|, \varphi(\boldsymbol{x})) \equiv(r, \varphi) .
$$

Let $F(\boldsymbol{x})$ denote a finite complex-valued narrowband multipath field in a region of interest $\|x\| \leqslant R$, for some finite range $R$, generated by sources and scatterers external to that region. ${ }^{2}$ In particular, we assume that all sources exist outside some radius $R_{s}>R$. Then, $F(x)$ satisfies the Helmholtz or reduced wave equation (in the region of interest) [11]

$$
\triangle F(\boldsymbol{x})+k^{2} F(\boldsymbol{x})=0, \quad k \triangleq 2 \pi / \lambda, \quad\|\boldsymbol{x}\| \leqslant R
$$

where $\triangle$ is the Laplacian and $\lambda$ is the wavelength. The time harmonic solution to the related full wave equation is then $F(\boldsymbol{x}, t)=F(\boldsymbol{x}) e^{-i \omega t}$, where $i=\sqrt{-1}, \omega=2 \pi f$, and $f$ is the frequency [11]. Since (1) is a linear partial differential equation (PDE), we see immediately that valid multipath fields in a source-free region of interest are constrained to lie in a linear subspace given by the nullspace of the operator $M \triangleq \triangle+k^{2} I$ ( $I$ is the identity operator). That is, if $F_{1}$ and $F_{2}$ are any two solutions of (1), then so is $\zeta_{1} F_{1}+\zeta_{2} F_{2}$ with $\zeta_{1}$ and $\zeta_{2}$ being scalars.

\footnotetext{
${ }^{2}$ More generally, the region could be any shape. This paper considers circular regions, because this is one of the cases where rigorous analytic bounds are possible. How more general shapes can be treated in an analogous way is not explicitly treated in this paper.
} 
In the framework of continuous sampling in space, we consider the wavefield $F(\boldsymbol{x})$ at point $\boldsymbol{x}$, without explicitly assuming an $N$-element array of receiver antennas (that could be located at points $\boldsymbol{x}_{1}$ to $\left.\boldsymbol{x}_{N}\right)$. We will quantify how $F(\boldsymbol{x})$ essentially has a finite dimensionality and richness. Since the treatment considers wavefields, regardless of the number of antenna elements or their placement, it can be concluded that that no matter how antenna elements are placed, the scattering has a limited richness.

Two broad classes of representations of the solution to (1) will be considered. The first class is based on plane wave synthesis and is considered in Section II-A. The second class is based on orthogonal solutions to the wave equation and is considered in Section II-B.

\section{A. Multiple Plane Wave Representation}

A standard multipath model involves modeling every distinct path explicitly as a plane wave. The plane wave model assumes the scattering to be in the far field, which primarily makes the analysis relevant for outdoor scenarios. In this model, the field is given by

$$
F(\boldsymbol{x})=\sum_{p} a_{p} e^{i k \boldsymbol{x} \cdot \widehat{\boldsymbol{y}}_{p}}
$$

where the plane wave of index $p$ has complex amplitude $a_{p} \in \mathbb{C}$, the propagation direction is denoted by the unit vector $\widehat{\boldsymbol{y}}_{p} \equiv$ $\left(1, \varphi_{p}\right)$, and $\boldsymbol{x} \cdot \boldsymbol{y}$ denotes the scalar (dot) product between vectors $\boldsymbol{x}$ and $\boldsymbol{y}$. We interpret representation (2) as encoding the field with a countable number of pairs $\left\{a_{p}, \widehat{\boldsymbol{y}}_{p}\right\}$ enumerated by $p$. Representations similar to (2) appear in array sensor signal processing applications, such as in [15]. Typically, only a finite number of plane waves are considered, although distributed sources are considered in [16]-[18]. For example, [18] extended the classical Bello's work [19] and established the duality between continuous spatial direction dispersion and spatial selectivity. The spatial duality is analogous to the duality between delay dispersion and frequency selectivity or between Doppler frequency dispersion and time selectivity in wireless channels.

A ready generalization that subsumes (2) is the superposition of plane waves from all azimuth directions $\varphi$ as

$$
F(\boldsymbol{x})=\int_{0}^{2 \pi} a(\varphi) e^{i k \boldsymbol{x} \cdot \widehat{\boldsymbol{y}}} d \varphi
$$

where $\widehat{y} \equiv(1, \varphi)$ and $a(\varphi)$ is the complex-valued gain of scatterers as a function of the direction of arrival $\varphi$.

\section{B. Orthogonal Representation}

A more general representation than (3), which implicitly required that any sources be in the farfield, is now given. If we assume that the narrowband multipath field is generated by sources outside some radius $R_{s}$, then for $R_{s} \rightarrow \infty$, we can use the Jacobi-Anger expansion [11, p. 67] to represent the plane waves in (3) as

$$
e^{i k \boldsymbol{x} \cdot \widehat{\boldsymbol{y}}}=\sum_{n=-\infty}^{\infty} i^{n} J_{n}(k\|\boldsymbol{x}\|) e^{i n(\varphi(\boldsymbol{x})-\varphi)}
$$

where $J_{n}(\cdot)$ is the Bessel function of the first kind of integer order $n$ [20], [21]. In array signal processing applications, the Jacobi-Anger expression is used to expand the elements of array covariance matrix [15]. By substituting (4) into (3), we obtain

$$
F(\boldsymbol{x})=\sum_{n=-\infty}^{\infty} i^{n} \alpha_{n} J_{n}(k\|\boldsymbol{x}\|) e^{i n \varphi(\boldsymbol{x})}, \quad\|\boldsymbol{x}\| \leqslant R<R_{s}
$$

where $\alpha_{n} \in \mathbb{C}$ is the $n$th Fourier series coefficient of $a(\varphi)$ in (3) defined as

$$
\alpha_{n}=\int_{0}^{2 \pi} a(\varphi) e^{-i n \varphi} d \varphi
$$

and

$$
a(\varphi)=\sum_{n=-\infty}^{\infty} \frac{\alpha_{n}}{2 \pi} e^{i n \varphi} .
$$

This shows the relationship between the angular distribution of far-field sources $a(\cdot)$ in (3) and the coefficients $\left\{\alpha_{n}\right\}$ of the general expression (5). In (5), we identify a countable set of orthonormal basis functions over the 2-D disc of size $R$ as

$$
\Phi_{n}(\boldsymbol{x}) \triangleq i^{n} \frac{J_{n}(k\|\boldsymbol{x}\|)}{\sqrt{\mathcal{J}_{n}(R)}} \frac{e^{i n \varphi(\boldsymbol{x})}}{\sqrt{2 \pi}}, \quad n \in \mathbb{Z}, \quad\|\boldsymbol{x}\| \leqslant R
$$

where

$$
\mathcal{J}_{n}(R) \triangleq \int_{0}^{R} J_{n}^{2}(k r) r d r .
$$

The orthonormality is verified

$$
\int_{0}^{R} \int_{0}^{2 \pi} \Phi_{n}(\boldsymbol{x}) \Phi_{m}^{*}(\boldsymbol{x}) d \varphi r d r=\left\{\begin{array}{ll}
1 & m=n \\
0 & \text { otherwise }
\end{array} .\right.
$$

We can rewrite (5) in its orthonormal expansion

$$
F(\boldsymbol{x})=\sum_{n=-\infty}^{\infty} \sqrt{2 \pi \mathcal{J}_{n}(R)} \alpha_{n} \Phi_{n}(\boldsymbol{x}) .
$$

In comparison with (2) and (3), representation (11) encodes

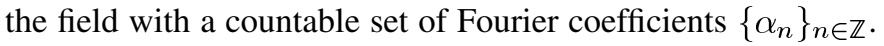
Moreover, the multipath field in (11) is represented as a superposition of a countable set of orthonormal basis functions $\left\{\Phi_{n}(x)\right\}_{n \in \mathbb{Z}}$, whereas (2) lacks a parsimonious property, since plane waves lack orthogonality.

The sequence $\left\{\alpha_{n}\right\}_{n \in \mathbb{Z}}$ in (5) and its statistical properties play a central role in this paper, as they provide, through truncation, an efficient or parsimonious parameterization of general narrowband multipath fields and allow studying their dimensionality or degrees of freedom.

\section{Random Multipath Fields}

Detailed information about scatterers that generate the multipath field $F(x)$ is usually limited. Therefore, it is reasonable to represent a multipath field $F(\boldsymbol{x})$ as a random process. Referring to (3), the scattering gain $a(\cdot)$ is random and so is $\alpha_{n}$ in (11). 
For mathematical tractability of the analysis, we assume uncorrelated scattering, which means that the random gains $a(\varphi)$ and $a\left(\varphi^{\prime}\right)$ at two distinct incident angles are uncorrelated from each other and the normalized APS is given by

$$
P(\varphi) \triangleq \frac{\mathcal{E}\left\{a(\varphi) a^{*}(\varphi)\right\}}{\int_{0}^{2 \pi} \mathcal{E}\left\{a(\varphi) a^{*}(\varphi)\right\} d \varphi}
$$

where $\mathcal{E}\{\cdot\}$ and $*$ denote expectation and complex conjugate, respectively. Using (6), (12), the uncorrelated scattering assumption, and following a few intermediate steps we find that

$$
\mathcal{E}\left\{\left|\alpha_{n}\right|^{2}\right\}=\int_{0}^{2 \pi} \mathcal{E}\left\{a(\varphi) a^{*}(\varphi)\right\} d \varphi .
$$

The normalized correlation between $\alpha_{n}$ and $\alpha_{m}$ is defined as

$$
\gamma_{m-n} \triangleq \frac{\mathcal{E}\left\{\alpha_{m} \alpha_{n}^{*}\right\}}{\mathcal{E}\left\{\left|\alpha_{n}\right|^{2}\right\}}=\int_{0}^{2 \pi} P(\varphi) e^{-i(m-n) \varphi} d \varphi .
$$

It is observed from (14) that $\gamma_{m-n}$ is, in fact, the $(m-n)$ th Fourier series coefficient of the normalized APS, $P(\varphi)$.

Using (3), the normalized spatial correlation function (SCF) of multipath fields $F\left(\boldsymbol{x}_{1}\right)$ and $F\left(\boldsymbol{x}_{2}\right)$ at points $\boldsymbol{x}_{1}$ and $\boldsymbol{x}_{2}$ is defined as [13]

$$
\begin{aligned}
\rho\left(\boldsymbol{x}_{2}, \boldsymbol{x}_{1}\right) \\
\quad=\frac{\mathcal{E}\left\{F\left(\boldsymbol{x}_{2}\right) F^{*}\left(\boldsymbol{x}_{1}\right)\right\}}{\mathcal{E}\left\{F\left(\boldsymbol{x}_{1}\right) F^{*}\left(\boldsymbol{x}_{1}\right)\right\}} \\
=\frac{\int_{0}^{2 \pi} \int_{0}^{2 \pi} \mathcal{E}\left\{a(\varphi) a^{*}\left(\varphi^{\prime}\right)\right\} e^{i k\left(\boldsymbol{x}_{2} . \widehat{\boldsymbol{y}}-\boldsymbol{x}_{1} . \widehat{\boldsymbol{y}}^{\prime}\right)} d \varphi d \varphi^{\prime}}{\int_{0}^{2 \pi} \int_{0}^{2 \pi} \mathcal{E}\left\{a(\varphi) a^{*}\left(\varphi^{\prime}\right)\right\} e^{i k\left(\boldsymbol{x}_{1} . \widehat{\boldsymbol{y}}-\boldsymbol{x}_{1} . \widehat{\boldsymbol{y}}^{\prime}\right)} d \varphi d \varphi^{\prime}} .
\end{aligned}
$$

Since we assume uncorrelated scattering, $\mathcal{E}\left\{a(\varphi) a^{*}\left(\varphi^{\prime}\right)\right\}$ in (16) is nonzero only for $\varphi=\varphi^{\prime}$. Therefore, the double integrals in (16) reduce to single integrals and upon using the APS definition in (12), $\rho\left(\boldsymbol{x}_{2}, \boldsymbol{x}_{1}\right)$ is simplified to

$$
\rho\left(\boldsymbol{x}_{2}, \boldsymbol{x}_{1}\right)=\rho\left(\boldsymbol{x}_{2}-\boldsymbol{x}_{1}\right)=\int_{0}^{2 \pi} P(\varphi) e^{i k\left(\boldsymbol{x}_{2}-\boldsymbol{x}_{1}\right) \cdot \widehat{\boldsymbol{y}}} d \varphi .
$$

Equation (17) shows that due to the uncorrelated scattering assumption, the SCF is only a function of the spatial separation between points $x_{2}$ and $x_{1}$ and therefore, is spatially stationary. Using the Jacobi-Anger expansion (4) for the plane waves in (17) yields

$$
\begin{gathered}
\rho\left(\boldsymbol{x}_{2}-\boldsymbol{x}_{1}\right) \\
=\sum_{m=-\infty}^{\infty}\left[i^{-m} J_{m}\left(k\left\|\boldsymbol{x}_{1}\right\|\right) e^{-i m \varphi\left(\boldsymbol{x}_{1}\right)}\right. \\
\left.\cdot \sum_{n=-\infty}^{\infty} i^{n} \gamma_{n-m} J_{n}\left(k\left\|\boldsymbol{x}_{2}\right\|\right) e^{i n \varphi\left(\boldsymbol{x}_{2}\right)}\right] \\
=\sum_{m=-\infty}^{\infty} i^{m} \gamma_{m} J_{m}\left(k\left\|\boldsymbol{x}_{2}-\boldsymbol{x}_{1}\right\|\right) e^{i m \varphi_{21}}
\end{gathered}
$$

where the second equality is written using the summation theorem for Bessel functions [22, pp. 930, 931]. In (19), $\left\|x_{2}-x_{1}\right\|$ is the distance between vectors $\boldsymbol{x}_{2}$ and $\boldsymbol{x}_{1}$ and $\varphi_{21}$ is the angle of the vector that connects $\boldsymbol{x}_{1}$ to $\boldsymbol{x}_{2}$. The SCF in (17)-(19) will be used later in Section III-B and Section IV to find the dimensionality and richness of random multipath fields, respectively.

Before concluding this section, we reiterate that the assumption of spatially uncorrelated scattering, originally used in [19] for uncorrelated scattering in delay dispersion domain, enables a mathematically tractable analysis. However, the assumption of spatially uncorrelated scattering may be violated in practical situations, where electromagnetic rays reflect nonspecularly off scatterers and the received amplitudes and phases of paths with similar angles of arrivals become correlated. Recently, [23] proposed a physical model to represent spatially correlated scattering, which requires a 2-D Fourier expansion of the scattering APS, $P(\varphi)$. It is shown that a scattering correlation with Gaussian density and the standard deviation of 2 degrees has modest and mostly negligible effects on the SCF, whereas for a randomly generated angular correlation, the difference cannot be neglected. The actual effect of correlated scattering on the dimensionality of multipath fields is still unknown. Our conjecture is that it could only reduce the dimensionality and not increase it. Hence, the results of this paper may serve as an upper bound on the available degrees of freedom. Quantifying the effects of correlated scattering on multipath dimensionality is an open problem.

\section{Dimensionality OF Multipath FieldS}

Multipath fields are usually observed in a limited region in space and, as such, the degree to which one can determine the effects of the multipath field is also limited. In the following sections, we will see that one needs to consider explicitly the region in space when considering concepts such as dimensionality and richness.

In Section II-B, the multipath field $F(\boldsymbol{x})$ was represented by a countable, but infinite sum of orthogonal modes $\left\{\alpha_{n} \Phi_{n}(\boldsymbol{x})\right\}_{n \in \mathbb{Z}}$ in (11). In this section, we define the dimensionality of multipath by the number or cardinality of effective modes that essentially build the field. To this end, we define the truncated field $F_{N}(\boldsymbol{x})$ by selecting the first $2 N+1$ indexed coefficients of $F(\boldsymbol{x})$

$$
F_{N}(\boldsymbol{x}) \triangleq \sum_{n=-N}^{N} \sqrt{2 \pi \mathcal{J}_{n}(R)} \alpha_{n} \Phi_{n}(\boldsymbol{x}) .
$$

As will be shown in the following sections, although all $\alpha_{n}$ coefficients in the synthesis of multipath field in (11) have the same variance [refer to (13)], it is still possible to accurately truncate the field with a carefully chosen truncation length $N$. Equivalently, the truncated field in (20) is synthesized as if the Fourier series coefficients $\alpha_{n}$ were zero for $|n|>N$. The question is how to choose $N$ so that the truncated field represents the actual field $F(\boldsymbol{x})$ within a given region and with a prescribed accuracy. 
Our approach consists of two parts. In Section III-A, we find an upper bound on the normalized truncation error of the multipath field as a function of the truncation length $N$. This is carried out for an arbitrary multipath (subject to satisfying conditions discussed in Section II), without explicitly considering its random nature. Hence, the field is assumed to be deterministic. From the truncation error upper bound in Section III-A we conclude that the effective dimensionality of multipath is directly related to the radius of the 2-D region, to which the field is coupled. Then in Section III-B, we find a universal upper bound on the normalized MSE of multipath field truncation, which takes multipath randomness into account. Considering the truncation MSE upper bound consistently predicts the same dimensionality for random multipath fields as in Section III-A. This is expected, because the absolute upper bound in Section III-A applies for any multipath. However, the MSE upper bound bears a more physical significance, because: 1) it takes the stochastic random scattering into account by computing the average error; and 2) it signifies the normalized energy of truncation error, which is more physically meaningful than the absolute error. Moreover, considering truncation MSE of random multipath fields paves the way for the MMSE KL expansion of multipath in Section IV and for investigating the dimensionality of random fields with a limited angular power spread and for quantifying multipath richness.

\section{A. Arbitrary, Deterministic Multipath Fields}

Using (11) and (20), we define the normalized field truncation error of the multipath field, over a 2-D disk of radius $R$, as

$$
\varepsilon_{N}(R) \triangleq \frac{1}{\pi R^{2}} \int_{0}^{R} \int_{0}^{2 \pi} \frac{\left|F(\boldsymbol{x})-F_{N}(\boldsymbol{x})\right|}{\|a\|} d \varphi r d r
$$

where assuming far-field sources $R_{s} \rightarrow \infty,\|a\|$ is finite and defined to be

$$
\|a\| \triangleq \int_{0}^{2 \pi}|a(\varphi)| d \varphi<\infty .
$$

Based on (22) and referring to (3), the field intensity $|F(\boldsymbol{x})|$ is also upper bounded by $\|a\|$. The normalization in (21) provides a relative error satisfying properties of: 1) scale invariance, that the relative error is the same for $F(\boldsymbol{x})$ and $\gamma F(\boldsymbol{x})$ for complex scalar $\gamma \neq 0$; and 2) unit plane wave invariance, that normalization leaves a unit amplitude plane wave unchanged.

We now elaborate on the normalized truncation error in (21). Since $F_{N}(\boldsymbol{x})$ is the truncation of an exact series, the two fields $F(\boldsymbol{x})$ and $F_{N}(\boldsymbol{x})$ will be essentially indistinguishable at any point $\|\boldsymbol{x}\| \leqslant R$, provided that $N$ is appropriately chosen according to $\|x\|$. In mathematical terms

$$
\begin{aligned}
& \lim _{N \rightarrow \infty} \frac{\left|F(\boldsymbol{x})-F_{N}(\boldsymbol{x})\right|}{\|a\|}=0 \Leftrightarrow \\
& \forall \epsilon>0, \exists N_{0}: \frac{\left|F(\boldsymbol{x})-F_{N}(\boldsymbol{x})\right|}{\|a\|}<\epsilon \text { for } N>N_{0} .
\end{aligned}
$$

The first contribution of this paper is to precisely quantify the limit parameters and to show how $N_{0}$ may be chosen, so that the normalized truncation error is smaller than $\epsilon$, for any given $\epsilon$. More specifically, we will show that

$$
\begin{aligned}
& \frac{\left|F(\boldsymbol{x})-F_{N}(\boldsymbol{x})\right|}{\|a\|}<0.16127 e^{-\Delta} \text { for } \\
& \qquad N>\lceil e \pi\|\boldsymbol{x}\| / \lambda\rceil+\Delta, \quad \Delta \geqslant 0
\end{aligned}
$$

where $\lceil\cdot\rceil$ is the integer ceiling function. Therefore, in (23) and for any given $\epsilon$, one can find $\Delta \geqslant 0$ such that $0.16127 e^{-\Delta}<\epsilon$ and then, choose $N_{0}=\lceil e \pi\|\boldsymbol{x}\| / \lambda\rceil+\Delta$. Throughout the rest of the paper, the statement "the truncation error exponentially approaches zero for $N>\lceil e \pi\|\boldsymbol{x}\| / \lambda\rceil$ " is interpreted in the context of (23) and (24). We will show that the decaying property of the truncation error carries over to a 2-D disk of size $R$. In particular, the truncation error in (21) exponentially approaches zero for $N_{R}>\lceil e \pi R / \lambda\rceil$.

Now, consider the integrand in (21), which is written using (5)

$$
\begin{aligned}
\zeta_{N}(\boldsymbol{x}) & \triangleq \frac{\left|F(\boldsymbol{x})-F_{N}(\boldsymbol{x})\right|}{\|a\|} \\
& =\frac{1}{\|a\|}\left|\sum_{|n|>N} i^{n} \alpha_{n} J_{n}(k\|\boldsymbol{x}\|) e^{i n \varphi(\boldsymbol{x})}\right| \\
& \leqslant \frac{1}{\|a\|} \sum_{|n|>N}\left|\alpha_{n}\right|\left|J_{n}(k r)\right| \\
& \leqslant \sum_{|n|>N}\left|J_{n}(k r)\right| \\
& =2 \sum_{n>N}\left|J_{n}(k r)\right|
\end{aligned}
$$

where the second inequality follows from (6) and (22) $\left(\left|\alpha_{n}\right| \leqslant\|a\|\right)$ and the last equality follows from $J_{-n}(k r)=$ $(-1)^{n} J_{n}(k r)$. From (25), it is evident that the behavior of the truncation error depends on the properties of Bessel functions $\left|J_{n}(k r)\right|$ for sufficiently large $n$. We use the following bound for the Bessel function $\left|J_{n}(u)\right|[24$, p. 362]:

$$
\left|J_{n}(u)\right| \leqslant \frac{u^{n}}{2^{n} n !}, \quad z \geqslant 0
$$

to upper bound (25) as

$$
\zeta_{N}(\boldsymbol{x}) \leqslant 2 \sum_{n>N}\left|J_{n}(k r)\right| \leqslant 2 \sum_{n>N} \frac{(k r)^{n}}{2^{n} n !} .
$$

The following theorem shows quantitatively that, for a fixed $\boldsymbol{x}$, by taking the truncation depth $N$ large enough, we can make $\zeta_{N}(x)$ as small as desired.

Theorem 1: Relative Truncation Error Bound of the Multipath Field: A multipath field $F(\boldsymbol{x})$ generated by farfield sources, having representation (11), can be truncated to $|n| \leqslant N$ terms as in (20), where the normalized truncation error is upper bounded as

$$
\zeta_{N}(\boldsymbol{x}) \triangleq \frac{\left|F(\boldsymbol{x})-F_{N}(\boldsymbol{x})\right|}{\|a\|} \leqslant \eta e^{-\Delta}
$$

provided that the truncation length is chosen as

$$
N=\lceil e \pi\|\boldsymbol{x}\| / \lambda\rceil+\Delta .
$$

In the above, $\eta \approx 0.16127$ and $\Delta \in \mathbb{Z}^{+}$. 
Theorem 1 states that the relative truncation error is no more than $16.1 \%$ once $N$ equals the critical threshold $\lceil e \pi\|x\| / \lambda\rceil$, and thereafter decreases at least exponentially to zero as $N$ increases. In other words, a larger truncation depths only marginally increases the accuracy of the truncated field representation. See Appendix I for the proof. The significance of the critical truncation depth $\lceil e \pi\|\boldsymbol{x}\| / \lambda\rceil$ is that it is rigorously derived. Moreover, the truncation threshold is simple and robust enough to be used for engineering applications.

Now, we turn back to the definition of the normalized truncation error (21) over a disk of radius $R$. We note that $\zeta_{N}(\boldsymbol{x})$ in (27) only depends on $r=\|\boldsymbol{x}\| \leqslant R$. From Theorem 1, we conclude that for a fixed $r, \zeta_{N}$ exponentially decays to zero for $N>\lceil e \pi r / \lambda\rceil$. Therefore, we choose $N_{R}=\lceil e \pi R / \lambda\rceil+\Delta$, so that the truncation error at the outer edge of disc is bounded as $\zeta_{N_{R}}(R) \leqslant \eta e^{-\Delta}$. Since $N_{R} \geqslant\lceil e \pi r / \lambda\rceil$ for every $r \leqslant$ $R, \zeta_{N_{R}}(r) \leqslant \eta e^{-\Delta}$ for every $r \leqslant R$. As a result, $N_{R}=$ $\lceil e \pi R / \lambda\rceil+\Delta$ guarantees $\varepsilon_{N}(R)<\eta e^{-\Delta}$. This is summarized in the following corollary to Theorem 1 .

Corollary 1.1: Truncation of Multipath Fields in a 2-D Region: In the region $\|x\| \leqslant R$, the truncation of multipath field $F(\boldsymbol{x})$ to $F_{N_{R}}(\boldsymbol{x})$ in (20) results in a normalized absolute truncation error $\varepsilon_{N}(R)$ defined in (21). The truncation error is upper bounded as $\varepsilon_{N}(R)<\eta e^{-\Delta}$ for $N=N_{R}+\Delta$, where $\Delta \geqslant 0$ and the truncation depth $N_{R}$ is defined as

$$
N_{R} \triangleq\lceil e \pi R / \lambda\rceil .
$$

Theorem 1 complements the preliminary results in [8] by explicitly quantifying how the normalized truncation error $\zeta_{N}$ in (28) exponentially decreases as a function of excess truncation length $\Delta$, and by providing a more rigorous and detailed mathematical derivation of the bound in Appendix I. More specifically, in [8] the normalized truncation error $\zeta_{N}$ was bounded as

$$
\zeta_{N}(\boldsymbol{x}) \leqslant \sqrt{\frac{2}{(N+1) \pi}} \frac{\rho(N,\|\boldsymbol{x}\|)^{N+1}}{1-\rho(N,\|\boldsymbol{x}\|)}
$$

where

$$
\rho(N,\|x\|)=\frac{\pi e\|\boldsymbol{x}\| / \lambda}{N+1}
$$

and $N>\pi e\|\boldsymbol{x}\| / \lambda-1$. Equation (31) does not explicitly elaborate on how $\zeta_{N}(\boldsymbol{x})$ behaves for $N=\lceil e \pi\|\boldsymbol{x}\| / \lambda\rceil+\Delta$. Quantifying the truncation error $\zeta_{N}(\boldsymbol{x})$ in the form of (28) is one contribution of this paper. Corollary 1.1 is an another contribution of this paper, compared to [8], which explicitly defines and upper bounds the normalized truncation error over a 2-D disk of radius $R$.

Example: Fig. 1 compares the predicted relative truncation error bound in Theorem 1 with multipath simulation results. We have synthesized a large number of 2-D multipath fields according to the plane wave model (2) and computed and plotted the worst case relative error $\max _{\|\boldsymbol{x}\| \leqslant R}\left\{\zeta_{N}(\boldsymbol{x})\right\}$ in (25) over the circular region $\|x\| \leqslant 1 \lambda$ ( $\lambda$ is the wavelength). The parameter is the truncation depth $N$. Note that in the context of an antenna array for communications, this region is not so small since, for example, it would accommodate a linear array of 5 antennas or 13 antennas in a diamond configuration at $\lambda / 2$

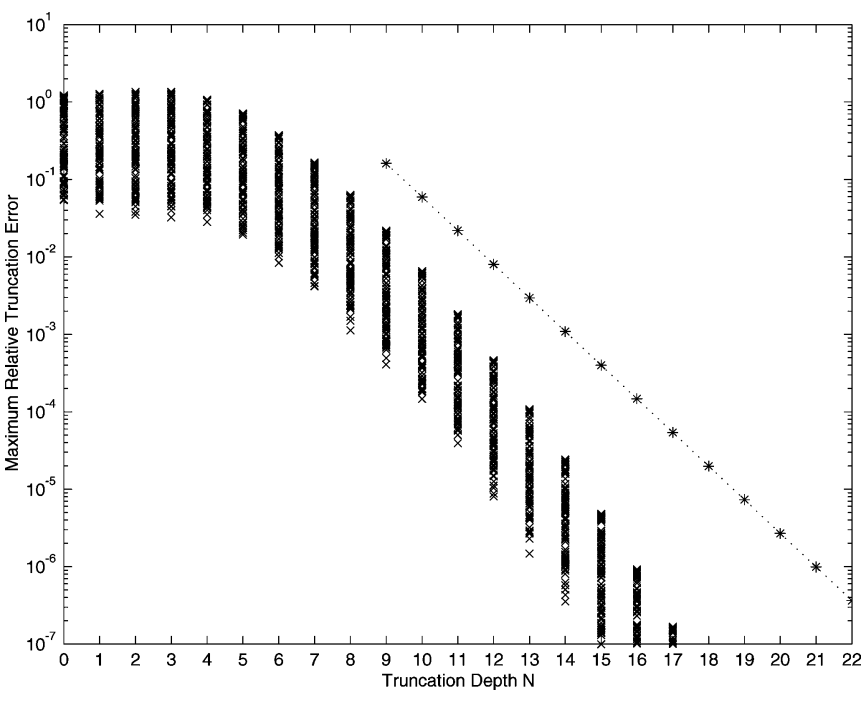

Fig. 1. Simulation results for the maximum relative error $\max _{\|\boldsymbol{x}\| \leqslant R}\left\{\zeta_{N}(\boldsymbol{x})\right\}$ versus the truncation order $N$ for a circular $2-\mathrm{D}$ region, $\|x\| \leqslant 1 \lambda$, for a large number of randomly generated 2-D multipath fields. Every trial value is marked with a cross. The stars define the analytic bound of (28) for $N=N_{R}+\Delta=$ $\lceil\pi e\rceil+\Delta \geqslant 9$, which is independent of the multipath field.

spacing. Fig. 1 summarizes the results of 50 trials, each field composed of $P \in\{1,2,5,10,50,100,200,500,1000\}$ plane waves. The directions of propagation $\varphi_{p}$ in (2) for the $P$ plane waves are uniformly chosen in the interval $[0,2 \pi)$. Therefore, the scattering is considered to be isotropic. However, when the number of plane waves is small, the resulted scattering is far from being dense. The complex amplitude $a_{p}$ of each plane wave was randomly selected from a uniform $[0,1]$ amplitude distribution and uniform $[0,2 \pi)$ phase distribution. It is evident that for all fields, the relative error decreases faster than exponentially with increasing $N$. In this figure, we have also plotted the bound (28). According to Theorem 1 and (30), for a 2-D region of size $\|x\| \leqslant 1 \lambda$ the truncation error exponentially decays if $N=N_{R}+\Delta=\lceil\pi e\rceil+\Delta \geqslant 9$. It is evident that the relative error bound is quite conservative and the actual relative error is of the order of $1 \%$ at $N_{R}=9$ (in comparison to our bound of approximately $16.1 \%$ ). The bound exponent does, however, well model the slope of the relative error at $N \cong N_{R}$. The fact that the slope is greater at higher truncation lengths can be inferred from the proof given in Appendix I [see (72)], but has not been reflected in Theorem 1 to simplify its statement. These experiments and similar experiments for regions of different sizes verify that one can bound the relative error independent of the multipath field where the bound depends only on the size of the region. Therefore, it is essentially impossible to distinguish between fields with relatively few paths and ones with 1000 or more paths (including diffuse multipath) for this size region. This example quantitatively confirms the well-known limitation that array aperture (size) imposes on resolving multipath fields.

\section{B. Random Multipath Fields}

In this section, we prove a universal bound on the truncation error of random multipath fields in the MSE sense. Since the multipath field is a random process, we compute the normalized truncation MSE over a 2-D disc with radius $R$, when the field in 
(11) is truncated to its first $2 N+1$ terms in (20). This is defined as

$$
\bar{\varepsilon}_{N}(R)=\frac{\int_{0}^{R} \int_{0}^{2 \pi} \mathcal{E}\left\{\left|F(\boldsymbol{x})-F_{N}(\boldsymbol{x})\right|^{2}\right\} d \phi r d r}{\int_{0}^{R} \int_{0}^{2 \pi} \mathcal{E}\left\{|F(\boldsymbol{x})|^{2}\right\} d \phi r d r} .
$$

Compared to (21), please note that the expectation operation is over all multipath fields in the numerator and a different energy normalization factor in the denominator is used. Although, upper bounding the absolute truncation error is often harder than deriving a bound for the truncation MSE, the truncation MSE is physically more meaningful. This is because it evaluates the normalized average energy of truncation error. The exact decaying behavior of (33) cannot be directly inferred from Theorem 1. As will be seen in Theorem 2, the truncation MSE upper bound decays even faster than (28) with the excess truncation length $\Delta$ and, hence, strengthens the physical significance of the results. Moreover, the truncation MSE will upper bound the truncation MMSE in the KL expansion in Section IV. Therefore, studying the truncation MSE is nontrivial and useful. We use (11), (20), the orthonormality of basis functions $\Phi_{n}(\boldsymbol{x})$ in (10), and the definition of $\mathcal{J}_{n}(R)$ in (9) to write the MSE in (33) as

$$
\bar{\varepsilon}_{N}(R)=\frac{\int_{0}^{R} \sum_{|n|>N} \mathcal{E}\left\{\left|\alpha_{n}\right|^{2}\right\} J_{n}^{2}(k r) r d r}{\int_{0}^{R} \sum_{n} \mathcal{E}\left\{\left|\alpha_{n}\right|^{2}\right\} J_{n}^{2}(k r) r d r} .
$$

From (13) it is observed that the expectation $\mathcal{E}\left\{\left|\alpha_{n}\right|^{2}\right\}$ is independent of index $n$, which yields

$$
\begin{aligned}
\bar{\varepsilon}_{N}(R) & =\frac{\int_{0}^{R} \sum_{|n|>N} J_{n}^{2}(k r) r d r}{\int_{0}^{R} \underbrace{\sum_{n} J_{n}^{2}(k r)}_{1} r d r} \\
& =\frac{\int_{0}^{R} \sum_{|n|>N} J_{n}^{2}(k r) r d r}{(1 / 2) R^{2}}
\end{aligned}
$$

where the summation in the first denominator is equal to one according to the summation theorem for Bessel functions [22, pp. 930, 931]. To find an upper bound for (35), we use the same bound for the Bessel functions given in (26). The following theorem provides a universal upper bound for the MSE in (35).

Theorem 2: Universal MSE Upper Bound for the Truncation of 2-D Random Multipath Fields: A random multipath field $F(\boldsymbol{x})$ generated by far-field sources, having representation (11), can be truncated to $|n| \leqslant N$ terms as in (20), with the normalized truncation MSE given in (35), upper bounded as

$$
\bar{\varepsilon}_{N}(R) \leqslant \sigma e^{-2 \Delta}
$$

provided that $N$ is chosen as $N=\lceil e \pi R / \lambda\rceil+\Delta$ and $\Delta \in \mathbb{Z}^{+}$. In (36), $\sigma=0.0093$.

The proof is provided in Appendix II. We note that in the derivation of multipath truncation MSE in (33)-(36), we did not assume anything about the multipath $\mathrm{SCF}, \rho\left(\boldsymbol{x}_{2}-\boldsymbol{x}_{1}\right)$, or equivalently about the APS, $P(\phi)$ in (17). Therefore, (36) is a universal bound on multipath truncation MSE, regardless of mul- tipath scattering spatial correlation. Therefore, no matter how random the scatter is, the field can be truncated to $2 N+1$ terms in (20) with an upper bound on MSE given in (36). In other words, $2 N+1$ is an upper bound on the effective richness of random multipath fields in the MSE sense.

So far, we have observed that the truncation of multipath fields results in an exponentially decaying error both in the absolute error and in the MSE sense for the truncation depth $N \geqslant$ $\lceil e \pi R / \lambda\rceil$ (Theorems 1 and 2 ), where the actual number of terms in (20) to represent the field is $2 N+1$. As was observed in Fig. 1, the above truncation length is quite conservative. We are now in a position to provide the following definition for the dimension of multipath fields.

Definition 1: Universal Bound on the Dimension of 2-D Random Multipath Fields: For a circular region in space given by $\|x\| \leqslant R$, the effective dimension of a 2-D multipath field is given by

$$
\mathcal{D}_{R}^{2 \mathrm{D}} \triangleq 2\lceil e \pi R / \lambda\rceil+1 \approx\lceil 17.079 R / \lambda\rceil+1 .
$$

Here, we summarize a few observations based on the above definition.

- The dimension, $\mathcal{D}_{R}^{2 \mathrm{D}}$ increases with the linear size of the region $R / \lambda$ (in wavelengths), not with the area of the region.

- The dimension increases with the precision required. Dimension (37) actually specifies a threshold effect, where the truncation error is small (in the sense of Theorems 1 and 2 and Corollary 1.1) and is decreasing with an exponential rate.

- It is sufficient to use only $\mathcal{D}_{R}^{2 \mathrm{D}}$ of the $\alpha_{n}$ in (11) to effectively encode any field within a distance $R$ of the origin.

In the following two subsections, we interpret the finite dimensionality of multipath fields in the context of spatial sampling and multiple antenna communication systems.

\section{Spatial Sampling}

If an actual multipath field $F(\boldsymbol{x})$ can be expressed exactly in the form (20) for some $N$, then we refer to the multipath field as being mode-limited. Hence, we have an exact sampling theory analogous to the sampling theory of time harmonic functions. That is, $2 N+1$ appropriately chosen sampling points in space are sufficient to completely determine $F(\boldsymbol{x})$ in the 2-D region.

For a general field $F(\boldsymbol{x})$ restricted to the region $\|x\| \leqslant R$, our theory implies that $F(\boldsymbol{x})$ is essentially mode-limited within $\|\boldsymbol{x}\| \leqslant R$ and, thereby, is well represented by a limited number of spatial samples (equal to the dimension). The point to be made is that a sufficiently regular multipath field ${ }^{3}$ has an intrinsic spatial forgetting which gives it a natural parsimonious representation in terms of the lower-order terms in the expansion (11).

\section{Number and Placement of Receiver Antennas}

We can interpret the finite dimensionality of multipath fields in the context of multiple antenna communication sys-

\footnotetext{
${ }^{3}$ Sufficiently regular in the sense of this paper means that the multipath field is generated by discrete or distributed farfield sources. The generalization to nearfield sources requires a more refined argument which is beyond the scope of this paper, but nonetheless the general conclusion remains.
} 
tems. Consider $Q$ antennas in a region $\|\boldsymbol{x}\| \leqslant R$ at distinct locations $\boldsymbol{x}_{1}, \boldsymbol{x}_{2}, \ldots, \boldsymbol{x}_{Q}$ that are sampling a multipath field $F(\boldsymbol{x})$ for communication purposes, such as multiple-input multiple-output (MIMO) systems [3], [4]. Since $F(\boldsymbol{x})$ is well modeled by $F_{N}(\boldsymbol{x})$ with $\mathcal{D}_{R}^{2 \mathrm{D}}$ terms given in (37), we can regard up to $Q-\mathcal{D}_{R}^{2 \mathrm{D}}$ of the antennas as superfluous-that is, any number of antennas in the region $\|\boldsymbol{x}\| \leqslant R$ beyond the dimension of the region, $\mathcal{D}_{R}^{2 \mathrm{D}}$ provide little or no additional information, depending on how wisely the antennas are spaced. This guides how densely we can usefully populate space with antennas. For small spatial regions, $\mathcal{D}_{R}^{2 \mathrm{D}}$ is small and the asymptotic analysis and predictions about MIMO capacity enhancements should be cautiously interpreted.

It remains an open problem to determine the best placement of a given number of antenna elements in a given area (or volume) to maximize the capacity or minimize other system-related cost functions. However, some preliminary results for optimizing the capacity of MIMO systems are available in [25].

\section{E. Extension to 3-D Multipath Fields}

After studying the dimensionality of 2-D multipath fields, we are interested to find how the results are extended to the 3-D case. For this purpose one needs to consider the 3-D equivalent of the 2-D Helmholtz equation in (1) for a spherical region of radius $R / \lambda$ wavelengths, or $\|x\| \leqslant R$. The solution to 3-D Helmholtz equation is more mathematically involved than (5) and is given by

$$
F(\boldsymbol{x})=4 \pi \sum_{n=0}^{\infty} i^{n} j_{n}(k\|\boldsymbol{x}\|) \sum_{m=-n}^{n} \alpha_{n}^{m} Y_{n}^{m}(\widehat{\boldsymbol{x}}),\|\boldsymbol{x}\| \leqslant R
$$

where $\alpha_{n}^{m} \in \mathbb{C}$ are constants independent of position, and

$$
j_{n}(z) \triangleq \sqrt{\frac{\pi}{2 z}} J_{n+1 / 2}(z)
$$

are the spherical Bessel functions, and

$$
Y_{n}^{m}(\widehat{\boldsymbol{x}}) \triangleq \sqrt{\frac{2 n+1}{4 \pi} \frac{(n-|m|) !}{(n+|m|) !}} P_{n}^{|m|}(\cos \theta) e^{i m \varphi}
$$

are the spherical harmonic functions, which are expressed in terms of the associated Legendre polynomials $P_{n}^{m}(\cos \theta)$. In (40), $\theta$ is the elevation angle and $\varphi$ is the azimuth angle, as before. The 3-D field in (38) is encoded with the countable set $\left\{\alpha_{n}^{m}\right\}$, which are 3-D counterparts of $\alpha_{n}$ in (5). Also, $Y_{n}^{m}$ and $Y_{q}^{p}$ are orthogonal for $m \neq p$ or $n \neq q$. It was shown in [26] that the first summation in (38) can be truncated to $N$ terms as

$$
F_{N}(\boldsymbol{x})=4 \pi \sum_{n=0}^{N} i^{n} j_{n}(k\|\boldsymbol{x}\|) \sum_{m=-n}^{n} \alpha_{n}^{m} Y_{n}^{m}(\widehat{\boldsymbol{x}})
$$

where for $N=\lceil\pi R e / \lambda\rceil+\Delta$ the normalized absolute truncation error is bounded as

$$
\begin{aligned}
\varepsilon_{N}(\boldsymbol{x}) & \leqslant \sum_{n>N}(2 n+1)\left|j_{n}(k\|x\|)\right| \\
& \leq \sqrt{\pi} \sum_{n>N} \frac{(\pi\|x\| / \lambda)^{n}}{\Gamma(n+1 / 2)} \leqslant v e^{-\Delta}
\end{aligned}
$$

where $v \approx 0.67848$ and $\Gamma(\cdot)$ is the Gamma function. The proof is structurally similar to the proof of Theorem 1 and hinges on the asymptotically decaying behavior of spherical Bessel functions in (42) and (43). More details about characterization of 3-D multipath fields can be found in [26].

We note that by truncating $F(\boldsymbol{x})$ to $N$ terms in (41), the number of significant $\left\{\alpha_{n}^{m}\right\}$ coefficients is $(N+1)^{2}$. Hence, we can assert what follows.

Definition 2: Dimensionality of 3-D Multipath Fields: For a spherical region in space given by $\|x\| \leqslant R$, the effective dimensionality of 3-D multipath is given by

$$
\mathcal{D}_{R}^{3 \mathrm{D}} \triangleq(\lceil e \pi R / \lambda\rceil+1)^{2} \approx 72.923(R / \lambda)^{2} .
$$

In summary, $\mathcal{D}_{R}^{3 \mathrm{D}}$ given in (44) bounds the spatial dimensionality of 3-D multipath and increases quadratically with $R / \lambda$, not with the volume of the region. For the sphere, the dimensionality scales with the surface area. It is sufficient to use only $\mathcal{D}_{R}^{3 \mathrm{D}}$ of $\alpha_{n}^{m}$ in (38) to effectively encode any field within a distance $R$ of the origin.

\section{F. Dimensionality Results and Huygen's Principle}

Before concluding this section, we comment on the consistency of the dimensionality results obtained here with Huygen's principle [11, p. 53, p. 176]. In electromagnetic theory, Huygen's principle states that given the tangential components of the electric and magnetic fields on a surface enclosing a radiation source, the field anywhere outside the surface can be determined without the need to know the source distribution inside the surface. According to Huygen's principle, each point of an advancing wave front is, in fact, the center of a fresh disturbance and the source of a new train of waves. With Huygen's principle in mind, we can say that in the 2-D wave propagation, the electromagnetic field incident on the receiver can be determined from the field distribution on a circular ring enclosing the source. Likewise, in the 3-D wave propagation, the wave front incident on the receiver can be determined from the field distribution on the surface of a sphere enclosing the source. Hence, the fact that 2-D/3-D field dimensionality depends on the radius/area of the region is consistent with Huygen's principle.

\section{KARHUNEN-LOÈVE EXPANSION OF RANDOM MULTIPATH FIELDS: MULTIPATH RICHNESS}

In Section III, we found an upper bound on the MSE of multipath field truncation error when the field is represented by the natural choice of orthonormal basis in (11). We showed that the essential dimensionality of 2-D multipath, which is observed in a disk with radius $R$, is $2 N+1=2\lceil\pi R e / \lambda\rceil+1$, regardless of stochastic scattering characteristics. Since accurate truncation of multipath to $2 N+1$ terms applies for any (and every) farfield multipath, $2 N+1$ serves as an upper bound for multipath dimensionality. But, does $2 N+1$ truly predict the dimensionality of a random multipath field with a specific spatial correlation function (SCF)? Of particular interest is the case where multipath power has a limited angular power spread. It is generally known that a limited angular power spread makes the multipath process correlated and hence, reduces its degrees 
of freedom. However, quantification of the relation between the angular power spread and multipath dimensionality, which is based on the general wave propagation and stochastic scattering theory deserves further investigation.

The treatment of multipath fields using the orthonormal basis in (11) is insufficient for analyzing the effects of limited angular power spread on multipath dimensionality. In fact, $\left\{\alpha_{n}\right\}_{n \in \mathbb{Z}}$ that encode multipath in (11) may be correlated random variables, as defined in (12)-(19). While multipath truncation to $2 N+1$ terms in Section III is universally applicable to any multipath field, regardless of correlation in $\left\{\alpha_{n}\right\}_{n \in \mathbb{Z}}$, a customized model should allow the optimal, maximally parsimonious representation of a particular field with a given SCF. With a fixed truncation length, the truncation error will be minimum when the multipath field is truncated in its optimal representation, or the MMSE truncation. Alternatively, with a fixed truncation error, the optimal modeling of multipath allows the minimum truncation length. For 2-D fields with a limited angular power spread, multipath dimensionality will be often much less than the predicted $2 N+1$.

In this section, we first define the optimal representation of random multipath fields and then, use this definition to quantify the notion of multipath richness. The Karhunen-Loève (KL) expansion is a widely-used signal processing model for the optimal representation of stochastic processes in time domain. The KL expansion takes away the redundant correlation of a random process and allows its representation with the minimum number of uncorrelated terms [14]. Here, we apply the KL expansion for the representation of stochastic multipath fields in spatial domain. The objective is to find the maximally parsimonious truncation of a random field $F(\boldsymbol{x})$ and the effects of limited angular power spread on multipath dimensionality. The KL modeling of multipath is a novel means of defining multipath richness that complements and confirms previous approaches in [10] and [12].

Based on the KL theory, the optimal expansion of a random multipath field $F(x)$ in the region $\|x\| \leqslant R$ may be written as

$$
F(\boldsymbol{x})=\sum_{n=0}^{+\infty} \sqrt{\lambda_{n}} \beta_{n} \Psi_{n}(\boldsymbol{x})
$$

where $\beta_{n}$ is a sequence of uncorrelated (white) random variables with unit variance, and $\lambda_{n} \geqslant 0$ and $\Psi_{n}(\boldsymbol{x})$ are the $n$th eigenvalue and eigenfunction of the SCF, respectively. That is, in the 2-D case

$$
\int_{0}^{R} \int_{0}^{2 \pi} \rho\left(\boldsymbol{x}_{2}-\boldsymbol{x}_{1}\right) \Psi_{n}\left(\boldsymbol{x}_{1}\right) d \varphi_{1} r_{1} d r_{1}=\lambda_{n} \Psi_{n}\left(\boldsymbol{x}_{2}\right)
$$

where $\rho\left(x_{2}-x_{1}\right)$ was given in (18) and $x_{1} \equiv\left(r_{1}, \varphi_{1}\right)$.

The major difference between the orthogonal representations of the random multipath field in (11) and in (45) is that the coefficients $\alpha_{n}$ in (11), regarded as random variables, may be correlated with covariance coefficients given in (14), whereas all correlation is taken away from $\beta_{n}$ in (45). Moreover, the orthonormal functions $\Psi_{n}(\boldsymbol{x})$ in the KL expansion (45) are customized for a particular multipath field with a given SCF, whereas the closed-form, orthonormal functions $\Phi_{n}(\boldsymbol{x})$ in the expansion (11) are universally applicable for any multipath field. While the universal expansion of the 2-D field in (11) predicts a universal dimensionality of $2 N+1$, a specific multipath richness is predictable using the KL expansion. If we assume that (45) is arranged in descending order of eigenvalues, then the truncation of multipath field $F(\boldsymbol{x})$ to $2 N+1$ terms in (45) results in an MMSE [14] that is already upper bounded by the MSE in Theorem 2 for $N \geqslant\lceil e \pi R / \lambda\rceil$.

For isotropic scattering, with the APS given by $P(\varphi)=$ $1 / 2 \pi, \varphi \in[0,2 \pi)$, we have $\gamma_{0}=1$, and $\gamma_{n}=0$ for $n \neq 0$ [see (14)], and the coefficients $\left\{\alpha_{n}\right\}_{n \in \mathbb{Z}}$ in (11) are already uncorrelated. Therefore, $\Phi_{n}\left(\boldsymbol{x}_{1}\right)$ in (8) is the $n$th SCF eigenfunction and $\lambda_{n}=2 \pi \mathcal{J}_{n}(R)$ is its corresponding eigenvalue. In other words, the truncation MSE bound in Theorem 2 is tight for an isotropic multipath field, asserting it as the richest type of scattering.

For other types of scattering and according to (45), the number of effective terms that generate the field is directly related to the eigenvalue spread of SCF. Therefore, we study the characteristic of SCF eigenvalues. In Section IV-A, we define multipath richness based on SCF eigenvalues and also prove a lower bound on the maximum eigenvalue of $\rho\left(\boldsymbol{x}_{2}-\boldsymbol{x}_{1}\right)$. Section IV-B presents numerical results and compares the defined multipath richness in Section IV-A with those provided in [12].

\section{A. Multipath Richness}

We first review some of the important properties of SCF eigenvalues. The integral operator $A$ with kernel $\rho\left(\boldsymbol{x}_{2}-\boldsymbol{x}_{1}\right)$

$$
A f \triangleq \int_{0}^{R} \int_{0}^{2 \pi} \rho\left(\boldsymbol{x}_{2}-\boldsymbol{x}_{1}\right) f\left(\boldsymbol{x}_{1}\right) d \varphi_{1} r_{1} d r_{1}
$$

is symmetric, self-adjoint and compact [27]. Then, there exists at most countably many different eigenvalues of $A$. If $A$ has infinitely many eigenvalues $\lambda_{n}(0 \leqslant n<\infty)$, then [27, p. 191]

$$
\lim _{n \rightarrow \infty} \lambda_{n}=0
$$

We now show that the sum of all the eigenvalues of the kernel $\rho\left(\boldsymbol{x}_{2}-\boldsymbol{x}_{1}\right)$ is finite and equal to $\pi R^{2}$ in a 2-D disk of radius $R$. From [28, pp. 117, 118], it is known that the sum of eigenvalues is equal to the trace of kernel

$$
\sum_{n=0}^{\infty} \lambda_{n}=\int_{0}^{R} \int_{0}^{2 \pi} \rho\left(\boldsymbol{x}_{1}-\boldsymbol{x}_{1}\right) d \varphi_{1} r_{1} d r_{1}=\pi R^{2}
$$

since $\rho\left(\boldsymbol{x}_{1}-\boldsymbol{x}_{1}\right)=\rho(0)=1$ according to (12) and (17).

Now we are in a position to define multipath richness from the characteristics of SCF eigenvalues. In defining multipath richness, we use the fact that the total sum of eigenvalues in (45) is finite and propose the notion of significant eigenvalues.

Definition 3: Multipath Richness: Suppose that in the KL expansion of multipath field $F(x)$ given by (45) the eigenvalues are indexed in descending order (including multiple 
eigenvalues). The field is said to have richness $M$ when the following normalized eigenvalue residual is less than 0.01 .

$$
\frac{\sum_{m>M} \lambda_{m}}{\sum_{m=0}^{\infty} \lambda_{m}}<0.01
$$

In other words, multipath richness is defined to be $M$ when at least $99 \%$ of the multipath energy is contained in the first $M$ eigenvalues.

Now, we wish to study the properties of eigenvalues for multipath fields with a limited angular power spread in the APS. An APS with a limited angular power spread is called a directional APS. To this end, we assume the APS, $P(\varphi)$ in (12), to be nonzero and uniformly distributed in the azimuth angular interval $\varphi \in[-\Omega, \Omega)$, where $\Omega$ is a fraction of $\pi$. We denote this fraction by $\Omega=\pi / \tau(\tau>1)$. In short, we may refer to this directional APS as $P_{\Omega}(\varphi)=1 / 2 \Omega$.

The following theorem proves a lower bound on the largest eigenvalue of the SCF. It is noted that, according to (48), the sum of eigenvalues is fixed for a given disk radius $R$. Therefore, a lower bound on the largest eigenvalue upper bounds the sum of remaining eigenvalues. Hence, according to Definition 3, a larger lower bound on the maximum eigenvalue means reduced multipath richness.

Theorem 3: A Lower Bound on the Largest Eigenvalue of the Spatial Correlation Function: The largest eigenvalue of the spatial correlation function in (17) in a 2-D disk of size $R$ with a uniformly directional APS $P_{\Omega}(\varphi)$ in the range $\varphi \in[-\Omega, \Omega)$, where $\Omega=\pi / \tau, \tau>1$, is lower bounded by

$$
\lambda_{\max } \geqslant \max _{q \in \mathbb{Z}}\left\{2 \pi \sum_{m=-\infty}^{\infty} \mathcal{J}_{m}(R) \operatorname{sinc}^{2}(m \Omega-q \pi)\right\} .
$$

The proof is provided in Appendix III. It may be verified that the lower bound on the maximum eigenvalue increases with decreasing the angular power spread $\Omega$. This, in turn, results in a smaller multipath eigenvalue spread. Therefore, (50) quantifies, to some extent, the well-known qualitative relationship between eigenvalue spread and multipath richness. Equation (50) is useful in most situations, where closed-form expressions for multipath SCF eigenvalues do not exist.

Before concluding this section, we review a standard upper bound on the largest eigenvalue. For the 2-D case, the upper bound is written as $[27$, p. 86]

$$
\begin{aligned}
\lambda_{\max } & =\left\|\rho\left(\boldsymbol{x}_{2}-\boldsymbol{x}_{1}\right)\right\| \\
& \leqslant\left[\int_{0}^{R} \int_{0}^{R} \int_{0}^{2 \pi} \int_{0}^{2 \pi}\left|\rho\left(\boldsymbol{x}_{2}-\boldsymbol{x}_{1}\right)\right|^{2} d \varphi_{1} d \varphi_{2} r_{1} d r_{1} r_{2} d r_{2}\right]^{1 / 2}
\end{aligned}
$$

which, by using the definition of $\rho\left(\boldsymbol{x}_{2}-\boldsymbol{x}_{1}\right)$ in (18), is simplified to

$$
\lambda_{\max } \leqslant 2 \pi \sqrt{\sum_{m=\infty}^{\infty} \mathcal{J}_{m}(R) \sum_{n=-\infty}^{\infty} \mathcal{J}_{n}(R)\left|\gamma_{n-m}\right|^{2}} .
$$

In Section IV-B, the numerical comparison of the largest SCF eigenvalue with the lower and upper bounds shows that the derived lower bound in (50) is tight.

\section{B. Eigenvalue Analysis}

Before we present the eigenvalue analysis results, we briefly discuss two numerical calculation methods of SCF eigenvalues. Numerical eigenvalue computation is often inevitable, because closed-form expressions for SCF eigenvalues may not exist. More specifically, one needs to numerically solve (46), which is also known as the Fredholm equation [27, p. 209]. A known technique to solve equations similar to (46) is to accurately approximate the integrals on the left-hand side by a sum, and transform the problem to a finite-dimensional, matrix-based eigenequation [29, pp. 782-785]. For this purpose, a set of $P$ quadrature points and quadrature weights are required to sufficiently sample the integral. Suppose that the qth quadrature point and quadrature weight are denoted by $\boldsymbol{x}_{q}$ and $w_{q}$, respectively. Then, at each point $\boldsymbol{x}_{q},(46)$ is approximated as

$$
\sum_{p=0}^{P-1} w_{p} \rho\left(\boldsymbol{x}_{q}-\boldsymbol{x}_{p}\right) \Psi_{n}\left(\boldsymbol{x}_{p}\right)=\lambda_{n} \Psi_{n}\left(\boldsymbol{x}_{q}\right) .
$$

The computational complexity of simultaneously solving $P$ equations of the form (53) is $O\left(P^{3}\right)$ [29, p. 783]. Hence, a clever choice of quadrature points and weights is required to optimally sample the region of interest. As can be seen from (46), this involves selection of $P$ points in a 2-D circular region (or in a 3-D spherical region for 3-D multipath). For a large region radius $R$, populating the area (or volume) with enough quadrature points becomes problematic (as a rule of thumb, points at the outer edges of the region should be sampled with the separation no larger than $\lambda / 2$ ).

Here, we define an alternative approach for the numerical solution of SCF eigenvalues, which is based on the equivalence of representations of the multipath field $F(\boldsymbol{x})$ in (11) and (45). The main advantage of the proposed algorithm over the aforementioned quadrature-based numerical technique is that it does not try to explicitly solve (46), and as such, the selection of quadrature points is avoided. To see this, we combine (11) and (45) to write

$$
\begin{aligned}
F(\boldsymbol{x}) & =\sum_{n=-\infty}^{+\infty} \sqrt{2 \pi \mathcal{J}_{n}(R)} \alpha_{n} \Phi_{n}(\boldsymbol{x}) \\
& =\sum_{n=0}^{+\infty} \sqrt{\lambda_{n}} \beta_{n} \Psi_{n}(\boldsymbol{x}) .
\end{aligned}
$$

It is evident from (54) that the change of orthonormal basis from $\Phi_{n}(\boldsymbol{x})$ to $\Psi_{n}(\boldsymbol{x})$ whitens the weighted random sequence $\sqrt{2 \pi \mathcal{J}_{n}(R)} \alpha_{n}$ to obtain the uncorrelated random sequence $\sqrt{\lambda_{n}} \beta_{n}$ with variance $\lambda_{n}$. Let the covariance matrix of the sequence $\sqrt{2 \pi \mathcal{J}_{n}(R)} \alpha_{n}$ be denoted by $\Gamma \quad$ with elements $\mathcal{E}\left\{\sqrt{2 \pi \mathcal{J}_{m}(R)} \alpha_{m} \cdot \sqrt{2 \pi \mathcal{J}_{n}(R)} \alpha_{n}^{*}\right\}=$ $2 \pi \sqrt{\mathcal{J}_{m}(R) \mathcal{J}_{n}(R)} \gamma_{m-n}$. In Section III, we concluded that the first $2 N+1$ terms of $\sqrt{2 \pi \mathcal{J}_{n}(R)} \alpha_{n}$ adequately represent the field. We summarize the above discussion in the following algorithm to numerically obtain SCF eigenvalues $\lambda_{n}$. 


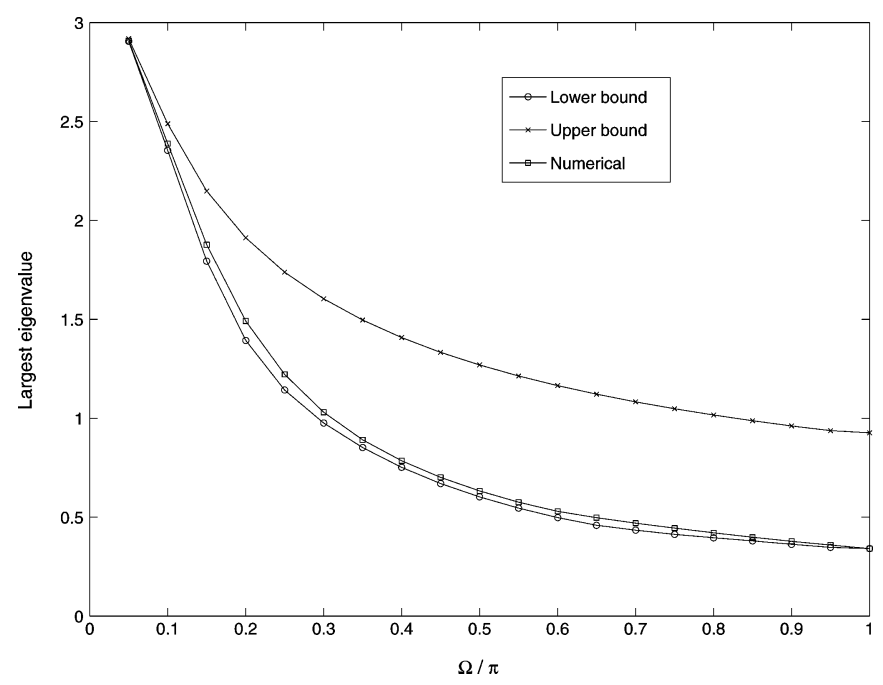

Fig. 2. The largest eigenvalue of the spatial correlation function as a function of APS spread $\Omega$. The eigenvalues are computed on a disk of radius $R=1 \lambda$. The lower bound is computed using (50) in Theorem 3. The upper bound is computed using (52). Also in the figure, the largest eigenvalue is calculated numerically using the algorithm in Section IV-B. The results show that the lower bound is very close to the actual eigenvalue and that multipath richness reduces with reducing $\Omega$. (Also see Table I.)

1) Based on Theorem 1 and Theorem 2, truncate the random sequence $\sqrt{2 \pi \mathcal{J}_{n}(R)} \alpha_{n}$ in (54) with truncation length $N \geqslant\lceil e \pi R \lambda\rceil$, to obtain the random vector

$$
\mathbf{v}=\left[\begin{array}{lll}
\sqrt{2 \pi \mathcal{J}_{-N}(R)} \alpha_{-N} & \cdots & \sqrt{2 \pi \mathcal{J}_{N}(R)} \alpha_{N}
\end{array}\right]^{T} .
$$

2) Using (14), form the covariance matrix for the random vector $\mathbf{v}$ defined as $\Gamma_{2 N+1}=\mathcal{E}\left\{\mathbf{v} \mathbf{v}^{H}\right\}$.

3) The eigenvalues of the covariance matrix $\Gamma_{2 N+1}$ give the first $2 N+1$ eigenvalues of the SCF $\rho\left(\boldsymbol{x}_{2}-\boldsymbol{x}_{1}\right)$.

Now, we present the lower bound, upper bound, and numerical results for the SCF largest eigenvalue for a set of directional scatterers. The results are shown in Fig. 2. The parameter is the angular power spread, $\Omega$, which varies from $\Omega / \pi=1 / 20$ (highly directional) to $\Omega / \pi=1$ (isotropic). The eigenvalues are computed on a disk with radius $R=1 \lambda$. The lower bound is computed using (50) in Theorem 3. The upper bound is computed using (52). Also in the figure, the largest eigenvalue is calculated numerically using the algorithm described above. It is clear from this figure that the proposed lower bound on the largest eigenvalue is tight. As $\Omega$ becomes smaller, the largest eigenvalue increases and multipath richness is reduced. Multipath richness for some typical values of $\Omega$ in this figure are calculated according to Definition 3 and are given in Table I. The results quantitatively confirm the qualitative speculations that reducing the multipath angular power spread would make the field more spatially correlated and reduce its richness.

Next, we present the numerical analysis of eigenvalues for three different APSs with angular power spread $\Omega / \pi=0.05$, 0.5 , and 1 . The eigenvalues are computed on a disk of size $R=1 \lambda$ using the algorithm described earlier. The results are shown in Fig. 3. From this figure, it is clear that the eigenvalue spread decreases with decreasing the angular power spread $\Omega$. Table I shows the predicted dimension or richness of multipath
TABLE I

DiMENSION OR RichNeSS OF MULTIPATH FIELDS STUdiED IN Figs. 2-3, Which is CAlCUlated Using Definition 3 AND ITS COMPARISON WiTH (55) [12]

\begin{tabular}{|c|c|c|c|c|c|}
\hline APS spread $\Omega / \pi$ & 0.05 & 0.25 & 0.5 & 0.75 & 1 \\
\hline Definition 3 & 3 & 6 & 9 & 12 & 15 \\
\hline $2\left\lceil\frac{\Omega e R}{\lambda}\right\rceil+1$ & 3 & 7 & 11 & 15 & 19 \\
\hline
\end{tabular}

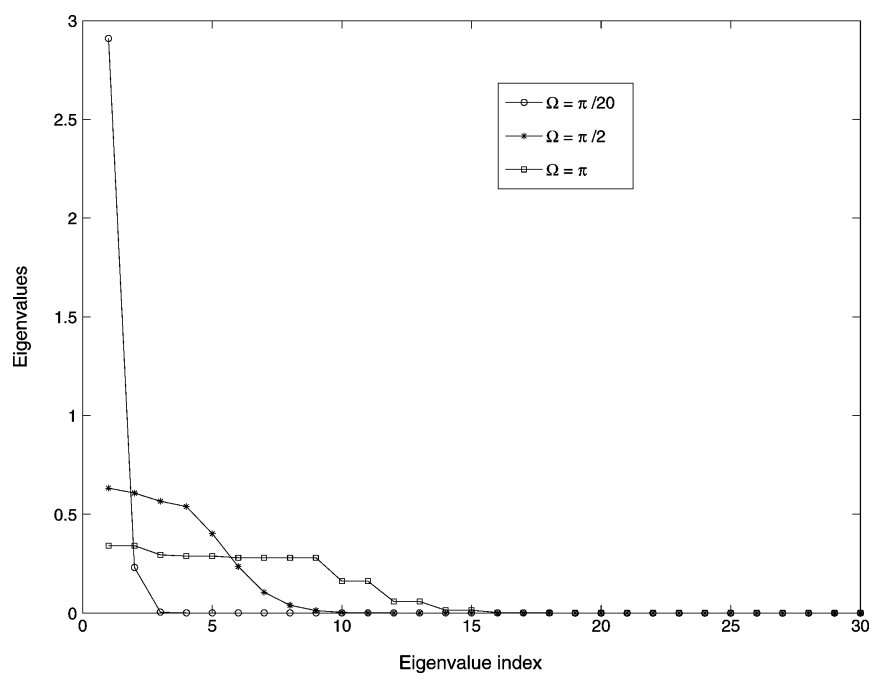

Fig. 3. Numerical eigenvalue analysis for three different APSs with spread $\Omega / \pi=0.05,0.5$, and 1 . The eigenvalues are computed on a disk of radius $R=1 \lambda$ using the algorithm in Section IV-B. The number of significant eigenvalues decreases with decreasing $\Omega$.

fields in Fig. 3 according to Definition 3, where the eigenvalue residual falls below 0.01 of the total $\pi R^{2}$. For comparison, we have also presented the predicted dimension of directional multipath fields in [12]. It was argued in [12] that the dimension of spatial multipath fields with a restricted angular power spread between $[-\Omega, \Omega)$ in a $2-D$ region of size $R$ is equal to

$$
2 M^{\prime}+1, \quad M^{\prime}=\left\lceil\frac{\Omega e R}{\lambda}\right\rceil .
$$

Compared to (37), the above dimension takes the restricted angular power spread into account. From Table I, it is observed that the calculated richness using Definition 3 is either identical to or below the predicted dimension in (55).

\section{CONCLUSION}

The degree to which a multipath field is resolvable in space depends on the size of space where the field is coupled to. For 2-D spatial regions of radius $R$, we proved that the number of effective multipath modes is limited by $2 N+1$, where $N=\lceil\pi e R / \lambda\rceil$. For 3-D spatial regions of radius $R$, the number of effective multipath modes is limited by $(N+1)^{2}$. We also defined random multipath richness based on the number of SCF eigenvalues in the KL expansion that capture $99 \%$ of the multipath energy. We showed that multipath richness is upper bounded by $2 N+1$ in the MMSE sense and the bound is achieved for isotropic multipath. By proving a lower bound on the largest SCF eigenvalue, we quantitatively verified the well-known reduction of multipath richness with reducing the 
angular power spread. Since the treatment in this paper considered wavefields, corresponding to the continuous sampling of space, it is concluded that random multipath scattering has an intrinsic limited richness, regardless of the number of receiver antenna elements or their placement. A possible extension of this paper would be to consider optimization of practical antenna arrangements to utilize the maximum available scattering richness for communication purposes. A practical application would be to optimize receiver antenna placements in MIMO systems to maximize channel information capacity for a given scattering environment.

\section{APPENDIX I \\ PROOF OF THEOREM 1}

Proof: For reference, (27) is repeated by defining $z \triangleq$ $\pi\|\boldsymbol{x}\| / \lambda$ and, hence, $k r=2 \pi / \lambda\|\boldsymbol{x}\|=2 z$

$$
\zeta_{N}(\boldsymbol{x}) \leqslant 2 \sum_{n>N}\left|J_{n}(2 z)\right| \leqslant 2 \sum_{n>N} \frac{z^{n}}{n !} \triangleq 2 R_{N}(z)
$$

Therefore, we find an upper bound on $R_{N}(z)$, which is expanded by changing the summation variable as

$$
\begin{aligned}
R_{N}(z) & =\sum_{n>N} \frac{z^{n}}{n !}=\exp (z)-\sum_{n=0}^{N} \frac{z^{n}}{n !} \\
& =\frac{z^{N+1}}{(N+1) !}\left(\sum_{n=0}^{\infty} \frac{(N+1) !}{(N+1+n) !} z^{n}\right) .
\end{aligned}
$$

Note that for integer $n \geqslant 0$, each term in the summation in (58) is upper bounded as

$$
\frac{(N+1) !}{(N+1+n) !} z^{n} \leqslant\left(\frac{z}{N+2}\right)^{n} .
$$

Hence, for integer $N \geqslant 0$ satisfying $N>z-2$, we can use the sum of geometric series to upper bound $R_{N}(z)$ in (58) as

$$
R_{N}(z) \leqslant \frac{z^{N+1}}{(N+1) !}\left(\frac{N+2}{N+2-z}\right) .
$$

Now, we use the Stirling lower bound, $n !>\sqrt{2 \pi n} n^{n} e^{-n}$, to write

$$
\frac{z^{N+1}}{(N+1) !} \leqslant \frac{1}{\sqrt{2 \pi(N+1)}}\left(\frac{e z}{N+1}\right)^{N+1} .
$$

Now, using the following exponential inequality

$$
\left(\frac{e z}{N+1}\right)^{N+1}=\left(1+\frac{e z-N-1}{N+1}\right)^{(N+1)} \leqslant e^{e z-N-1}
$$

we obtain the following upper bound on $R_{N}(z)$

$$
R_{N}(z) \leqslant \frac{e^{e z-N-1}}{\sqrt{2 \pi(N+1)}}\left(\frac{N+2}{N+2-z}\right) .
$$

To contain the exponential in (63), for a given $z$, consider selecting $N(z)=\lceil e z\rceil$. Then

$$
e z \leqslant N(z)<e z+1
$$

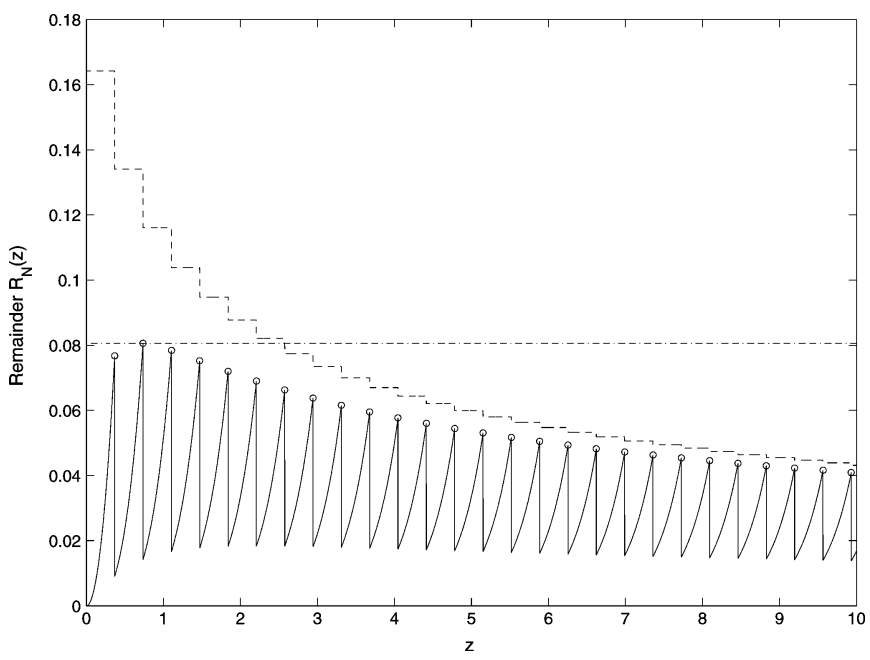

Fig. 4. The remainder $R_{N}(z)$ in (57) with $N=N(z) \equiv[e z\rceil$. The stepped curve is the bound given by (67) and the uniform bound corresponds to (68).

from which it can be shown that

$$
\begin{aligned}
\left(\frac{N(z)+2}{N(z)+2-z}\right) & \leqslant \frac{e}{e-1}=1.58197 \ldots \\
\exp (e z-N(z)-1) & \leqslant 1 / e=0.36787 \ldots .
\end{aligned}
$$

Using (65) and (66) in (63) yields

$$
R_{N(z)}(z) \leqslant \frac{1}{(e-1) \sqrt{2 \pi(N(z)+1)}}=\frac{0.23217 \ldots}{\sqrt{N(z)+1}} .
$$

Selecting $N(z)=\lceil e z\rceil$ implies that $R_{N(z)}(z)$ in (67) is a piecewise function of $z$ with local maxima at $z \in$ $\{1 / e, 2 / e, 3 / e, \ldots\}$, where $N(z)$ steps up to the next integer value. By searching over these local maxima we can use the exact expression (57) to obtain a uniform tight bound

$$
\begin{aligned}
R_{N(z)}(z) & \leqslant \max _{N} R_{N}(N / e)=R_{2}(2 / e) \\
& =\exp \left(\frac{2}{e}\right)-\left(1+\frac{2}{e}+\frac{2}{e^{2}}\right)=0.080635 \ldots
\end{aligned}
$$

which improves on (67) when $N(z) \leq 7$. Fig. 4 displays the truncation error bounds for $R_{N}(z)$ given in (67) and (68) as a function of $z$ and using $N=\lceil e z\rceil$.

When $N \geqslant N(z)$, which we refer to as the critical regime, we infer from (68) that $R_{N}(z) \leq 0.080635 \ldots$. Therefore, $\zeta_{N}(x) \leq 0.16127$ that establishes (28) for $\Delta=0$.

Now, we show that $R_{N}(z)$, for a fixed $z$, exponentially decreases as $N$ increases provided that $N>z-2$. First, we note that for a positive fixed $z, R_{N}(z)$ in (57) is decreasing with $N$. Using (58) we write the ratio $R_{N+\Delta}(z) / R_{N}(z)$ for $\Delta \geqslant 0$ as

$\frac{R_{N+\Delta}(z)}{R_{N}(z)}=\frac{z^{N+\Delta+1}}{(N+\Delta+1) !} \frac{(N+1) !}{z^{N+1}} \frac{\sum_{n=0}^{\infty} \frac{(N+\Delta+1) !}{(N+\Delta+1+n) !} z^{n}}{\sum_{n=0}^{\infty} \frac{(N+1) !}{(N+1+n) !} z^{n}}$.

We observe that for $\Delta \geqslant 0$

$$
\begin{aligned}
\frac{(N+\Delta+1) !}{(N+\Delta+1+n) !} & =\frac{1}{(N+\Delta+2) \cdots(N+\Delta+1+n)} \\
& \leqslant \frac{1}{(N+2) \cdots(N+1+n)} \\
& =\frac{(N+1) !}{(N+1+n) !} .
\end{aligned}
$$


Therefore, each term in the summation in numerator in (69) is smaller than each term in the summation in the denominator and hence, their ratio is less than 1 , which yields

$$
\begin{aligned}
\frac{R_{N+\Delta}(z)}{R_{N}(z)} & \leqslant \frac{z^{\Delta}}{(N+2)(N+3) \cdots(N+\Delta+1)} \\
& \leqslant\left(\frac{z}{N+2}\right)^{\Delta}=\left.\frac{1}{\alpha^{\Delta}}\right|_{\alpha=(N+2) / z} .
\end{aligned}
$$

Therefore, whenever $N>z-2$, we have $\alpha>1$ and the remainder $R_{N+\Delta}(z)$ decreases exponentially as $\Delta$ increases. In the critical regime, $N \geqslant\lceil e z\rceil$, this implies $\alpha>e$ and the exponential decrease is at least as fast as $\exp (-\Delta)$ by (72). Therefore, the truncation error upper bound for $N+\Delta=\lceil e z\rceil+\Delta$ is written as

$$
\zeta_{N+\Delta}(\boldsymbol{x}) \leqslant 2 R_{N+\Delta}(z) \leqslant 2 R_{N}(z) e^{-\Delta} \leqslant 0.16127 e^{-\Delta} .
$$

It is also easily verified that for all positive values of $z$ satisfying

$$
\frac{K-1}{e}<z \leqslant \frac{K}{e} \Rightarrow N=\lceil e z\rceil=K, \quad K \in \mathbb{N}
$$

the inequality $N>z-2$ is automatically satisfied and the assumptions throughout the proof are valid.

\section{APPENDIX II}

\section{PROOF OF THEOREM 2}

Proof: The proof of Theorem 2 is similar to the proof of Theorem 1. Referring to (35) and using (26), we first find an upper bound for the summation in the integral

$$
S_{N}(z) \triangleq \sum_{n=N+1}^{+\infty} J_{n}^{2}(2 z) \leqslant \sum_{n=N+1}^{+\infty} \frac{z^{2 n}}{(n !)^{2}}
$$

where $z \triangleq \pi\|\boldsymbol{x}\| / \lambda$. Using a similar procedure as in (57)-(59), we obtain

$$
S_{N}(z) \leqslant \frac{z^{2(N+1)}}{((N+1) !)^{2}} \frac{1}{1-\frac{z^{2}}{(N+2)^{2}}}
$$

for $N+2>z$. Now using the same steps as (61) and (62), the following bound is derived:

$$
S_{N}(z) \leqslant \frac{e^{2(e z-N-1)}}{2 \pi(N+1)} \frac{1}{1-\frac{z^{2}}{(N+2)^{2}}} .
$$

If we choose $e z \leqslant N=\lceil e z\rceil<e z+1$, we obtain

$$
S_{N}(z) \leqslant \frac{0.0249}{(N+1)} \text {. }
$$

We can further examine the bound on $S_{N}(z)$ given in (75) at critical points of the ceiling function $(z=k / e, k \in \mathbb{N})$ to obtain a universal upper bound

$$
S_{N}(z) \leqslant 0.004649
$$

by choosing $z=1 / e$ in (75). Similar to the steps in (69)-(72), it is possible to show that for $\Delta \geqslant 0, N=\lceil e z\rceil$, and $N+2>z$, the following ratio holds

$$
\frac{S_{N+\Delta}(z)}{S_{N}(z)} \leqslant e^{-2 \Delta} \text {. }
$$

Based on (78), it is possible to show that the truncation MSE in (35)

$$
\bar{\varepsilon}_{N}(R)=c \frac{\int_{0}^{R} 2 S_{N}(z) r d r}{1 / 2 R^{2}} \leqslant 0.009298 .
$$

Moreover, if we choose $N_{R}=\lceil e \pi R / \lambda\rceil+\Delta$, so that $S_{N}(z)$ at the outer edge of disk is bounded as $S_{N}(z) \leqslant 0.004649 e^{-2 \Delta}$, from (79) we conclude that $S_{N}(z) \leqslant 0.004649 e^{-2 \Delta}$ for every $r \leqslant R$. Therefore, $\bar{\varepsilon}_{N}(R) \leqslant 0.009298 e^{-2 \Delta}$, which completes the proof.

\section{APPENDIX III}

\section{PROOF OF THEOREM 3}

Proof: Before proceeding, we remember that according to the maximum eigenvalue property [27, p. 198] and by the definition of the operator norm [27] for the kernel $A=\rho\left(\boldsymbol{x}_{2}-\boldsymbol{x}_{1}\right)$

$$
\lambda_{\max }=\|A\| \geqslant\langle A g, g\rangle, \quad \forall g:\|g\|=1
$$

it is possible to find a lower bound on the maximum eigenvalue by arbitrarily choosing any function $g$ with the only condition being that $\|g\|=1$. In the following, we will obtain a close lower bound for $\lambda_{\max }$ by proper choice of $g$. Let the function $g(\boldsymbol{x})$ in the region $\|\boldsymbol{x}\| \leqslant R$ and $\boldsymbol{x} \equiv(r, \varphi)$ be expanded as

$$
g(\boldsymbol{x})=\sum_{p=-\infty}^{\infty} i^{p} b_{p} \frac{J_{p}(k r)}{\sqrt{\mathcal{J}_{p}(R)}} \frac{e^{i p \varphi}}{\sqrt{2 \pi}}
$$

where $\|g\|=1$ requires that $\sum_{p}\left|b_{p}\right|^{2}=1$. We write the inner product $\langle A g, g\rangle \triangleq \mu$ using (47), the definition of $\rho\left(\boldsymbol{x}_{2}-\boldsymbol{x}_{1}\right)$ in (18), and the definition of $g$ in (82), which yields

$$
\mu=2 \pi \sum_{m=-\infty}^{\infty} \sqrt{\mathcal{J}_{m}(R)} b_{m} \sum_{n=-\infty}^{\infty} \sqrt{\mathcal{J}_{n}(R)} b_{n}^{*} \gamma_{n-m} .
$$

Now, we use the definition of $\gamma_{n-m}$ in (14) for a directional APS $P_{\Omega}(\varphi)$ to write

$$
\mu=\frac{\pi}{\Omega} \int_{-\Omega}^{\Omega}\left|\sum_{m=-\infty}^{\infty} \sqrt{\mathcal{J}_{m}(R)} b_{m} e^{i m \varphi}\right|^{2} d \varphi .
$$

A set of orthonormal basis functions for the limited spread $[-\Omega, \Omega)=[-\pi / \tau, \pi / \tau)$ are $\left\{e^{i n \tau \varphi} / \sqrt{2 \Omega}\right\}_{n \in \mathbb{Z}}$. We expand the exponentials in (84) in terms of the new basis functions

$$
e^{i m \varphi}=\sum_{q=-\infty}^{\infty} \xi_{m, q} \frac{e^{i q \tau \varphi}}{\sqrt{2 \Omega}}
$$


where $\xi_{m, q} \triangleq \sqrt{2 \Omega} \operatorname{sinc}(m \Omega-q \pi)$. Replacing (85) into (84) and changing the summation order yields the following:

$$
\mu=\frac{\pi}{\Omega} \int_{-\Omega}^{\Omega}\left|\sum_{q=-\infty}^{\infty} \beta_{q} \frac{e^{i q \tau \varphi}}{\sqrt{2 \Omega}}\right|^{2} d \varphi
$$

where $\beta_{q}$ is defined as

$$
\beta_{q} \triangleq \sum_{m=-\infty}^{\infty} \sqrt{\mathcal{J}_{m}(R)} b_{m} \xi_{m, q}
$$

Now, using the orthonormality of the basis functions in (86) we write

$$
\mu=\frac{\pi}{\Omega} \sum_{q=-\infty}^{\infty} \beta_{q}^{2} .
$$

Since all the terms in the summation are nonnegative, the inner product $\mu$ is greater than every $(\pi / \Omega) \beta_{q}^{2}$ including its maximum $(\pi / \Omega) \beta_{\max }^{2} \triangleq(\pi / \Omega) \max _{q \in \mathbb{Z}}\left\{\beta_{q}^{2}\right\}$. To find the closest lower bound on $\mu$, we first use Cauchy's inequality to upper bound each term $\beta_{q}^{2}$ in (87)

$$
\begin{aligned}
\beta_{q}^{2} & =\left|\sum_{m=-\infty}^{\infty} \sqrt{\mathcal{J}_{m}(R)} b_{m} \xi_{m, q}\right|^{2} \\
& \leqslant\left(\sum_{m=-\infty}^{\infty} \mathcal{J}_{m}(R) \xi_{m, q}^{2}\right)\left(\sum_{m=-\infty}^{\infty} b_{m}^{2}\right)
\end{aligned}
$$

where the upper bound is achieved when

$$
b_{m, q}=c_{q} \sqrt{\mathcal{J}_{m}(R)} \xi_{m, q}
$$

and $c_{q}$ is a constant that is chosen

$$
c_{q}=\frac{1}{\sqrt{\sum_{m=-\infty}^{\infty} \mathcal{J}_{m}(R) \xi_{m, q}^{2}}}
$$

to normalize the function $g$ in (82) in the region $\|x\| \leqslant R$. Therefore, by choosing $b_{m, q}$ according to (90), each term $\beta_{q}^{2}$ is maximized as $\beta_{q}^{2}=\sum_{m=-\infty}^{\infty} \mathcal{J}_{m}(R) \xi_{m, q}^{2}$. And by definition

$$
\beta_{\max }^{2} \triangleq \max _{q \in \mathbb{Z}}\left\{\beta_{q}^{2}\right\}=\max _{q \in \mathbb{Z}} \sum_{m=-\infty}^{\infty} \mathcal{J}_{m}(R) \xi_{m, q}^{2}
$$

Finally, by combining (81), the definition of $\xi_{m, q}$ following (85), and (92) the following lower bound on the maximum eigenvalue is derived

$$
\begin{aligned}
& \lambda_{\max } \geqslant \mu \geqslant \frac{\pi}{\Omega} \beta_{\max }^{2}, \\
& \lambda_{\max } \geqslant \max _{q \in \mathbb{Z}}\left\{2 \pi \sum_{m=-\infty}^{\infty} \mathcal{J}_{m}(R) \operatorname{sinc}^{2}(m \Omega-q \pi)\right\} .
\end{aligned}
$$

\section{REFERENCES}

[1] T. S. Rappaport, Wireless Communications, Principles and Practice, 2nd ed. Upper Saddle River, NJ: Prentice-Hall, 2002.

[2] R. Kohno, "Spatial and temporal communication theory using adaptive antenna array," IEEE Pers. Commun. Mag., vol. 5, no. 1, pp. 28-35, Feb. 1998.

[3] I. E. Telatar, "Capacity of multiple-antenna Gaussian channels," Europ. Trans. Telecommun., vol. 10, pp. 585-595, Nov. 1999.

[4] G. J. Foschini and M. J. Gans, "On the limits of wireless communications in a fading environment when using multiple antennas," Wireless Pers. Commun., vol. 6, no. 2, pp. 311-355, 1998.

[5] J. P. Kermoal, P. E. Mogensen, S. H. Jensen, J. B. Andersen, F. Frederiksen, T. B. Sørensen, and K. I. Pedersen, "Experimental investigation of multipath richness for multi-element transmit and receive antenna arrays," in Proc. IEEE Veh. Technol. Conf., VTC-2000, Spring, Tokyo, Japan, May 2000, vol. 3, pp. 2004-2008.

[6] A. Lozano and C. Papadias, "Layered space-time receivers for frequency-selective wireless channels," IEEE Trans. Commun., vol. 50, no. 1, pp. 65-73, Jan. 2002.

[7] P. W. Wolniansky, G. J. Foschini, G. D. Golden, and R. A. Valenzuela, "V-BLAST: An architecture for realizing very high data rates over the rich-scattering wireless channel," in Proc. URSI Int. Symp. Sig. Syst. Elect., Thessaloniki, Greece, Sep.-Oct. 1998, pp. 295-300.

[8] H. M. Jones, R. A. Kennedy, and T. D. Abhayapala, "On dimensionality of multipath fields: Spatial extent and richness," in Proc. IEEE Int. Conf. Acoust., Speech Signal Process. (ICASSP), Orlando, FL, May 2002, pp. 2837-2840.

[9] R. A. Kennedy and T. D. Abhayapala, "Spatial concentration of wave-fields: Towards spatial information content in arbitrary multipath scattering," in Proc. 4th Australian Commun. Theory Workshop, AusCTW-2003, Melbourne, Australia, Feb. 2003, pp. 38-45.

[10] A. S. Y. Poon, R. W. Broderson, and D. N. C. Tse, "Degrees of freedom in multiple-antenna channels: A signal space approach," IEEE Trans. Inf. Theory, vol. 51, no. 2, pp. 523-536, Feb. 2005.

[11] D. Colton and R. Kress, Inverse Acoustic and Electromagnetic Scattering Theory, 2nd ed. New York: Springer-Verlag, 1998.

[12] G. Dickins, M. Williams, and L. W. Hanlen, "On the dimensionality of spatial fields with restricted angle of arrival," in Proc. IEEE Int. Symp. Inf. Theory (ISIT), Adelaide, Australia, Sep. 2005, pp. 1033-1037.

[13] P. S. Teal, T. D. Abhayapala, and R. A. Kennedy, "Spatial correlation for general distribution of scatterers," IEEE Signal Process. Lett., vol. 9, no. 10, pp. 305-308, Oct. 2002.

[14] R. G. Ghanem and P. D. Spanos, Stochastic Finite Elements: A Spectral Approach. New York: Courier Dover, 2003.

[15] T. Trump and B. Ottersten, "Estimation of nominal direction of arrival and angular spread using an array of sensors," Signal Process., vol. 50, no. 1-2, pp. 57-69, 1996.

[16] S. Valaee, B. Champagne, and P. Kabal, "On performance analysis for signaling on correlated fading channels," IEEE Trans. Signal Process. vol. 43, no. 9, pp. 2144-2153, Sep. 1995.

[17] M. Bengtsson and B. Ottersten, "Low-complexity estimators for distributed sources," IEEE Trans. Signal Process., vol. 48, no. 8, pp. 2185-2194, Aug. 2000

[18] B. H. Fleury, "First- and second-order characterization of direction dispersion and space selectivity in the radio channel," IEEE Trans. Inf. Theory, vol. 46, no. 6, pp. 2027-2044, Sep. 2000

[19] P. A. Bello, "Characterization of randomly time-variant linear channels," IEEE Trans. Commun. Syst., vol. CS-11, pp. 360-393, Dec. 1963.

[20] N. W. McLachlan, Bessel Functions for Engineers, 2nd ed. London, U.K.: Oxford Univ. Press, 1961.

[21] C. A. Coulson and A. Jeffrey, Waves: A Mathematical Approach to the Common Types of Wave Motion, 2nd ed. London, U.K.: Longman, 1977.

[22] I. S. Gradshteyn and I. M. Ryzhik, Table of Integrals, Series, and Products, 6th ed. San Diego, CA: Academic, 2000.

[23] T. Betlehem and T. D. Abhayapala, "Spatial correlation for correlated scatterers," in Proc. IEEE Int. Conf. Acoust., Speech, Signal Process. (ICASSP), Toulouse, France, May 2006, pp. IV-5-IV-8.

[24] M. Abramowitz and I. A. Stegun, Handbook of Mathematical Functions, 10th ed. New York: Dover, 1974.

[25] P. Sadeghi, T. D. Abhayapala, and R. A. Kennedy, "Directional random scattering MIMO channels: Entropy analysis and capacity optimization," in Proc. IEEE Int. Conf. Commun. (ICC), Istanbul, Turkey, Jun. 2006. 
[26] T. D. Abhayapala, T. S. Pollock, and R. A. Kennedy, "Characterization of 3D spatial wireless channels," in Proc. IEEE Veh. Technol. Conf. (VTC), Orlando, FL, Oct. 2003, pp. 123-127.

[27] G. Helmberg, Introduction to Spectral Theory in Hilbert Space. Amsterdam: North-Holland, 1969.

[28] A. V. Balakrishnan, Applied Functional Analysis. New York: Springer-Verlag, 1976.

[29] W. H. Press, B. P. Flannery, S. A. Teukolsky, and W. T. Vetterling, Numerical Recipes in C. Cambridge, U.K.: Cambridge Univ. Press, 1992.

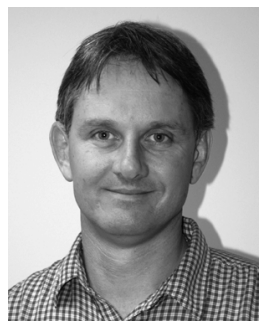

Rodney A. Kennedy (S'86-M'88-SM'01-F'05) received the B.E. degree from the University of New South Wales, Sydney, Australia, the M.E. degree from the University of Newcastle, and the Ph.D. degree from the Australian National University, Canberra.

He was with the Commonwealth Scientific and Industrial Research Organization (CSIRO) on the Australia Telescope Project for three years. He is Head of the Department of Information Engineering, Research School of Information Sciences and Engineering, Australian National University. His research interests are in the fields of digital signal processing, spatial information systems, digital and wireless communications, and acoustical signal processing.

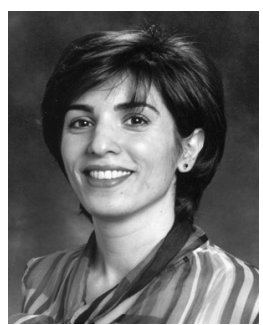

Parastoo Sadeghi (S'02-M'06) received the B.E. and M.E. degrees in electrical engineering from Sharif University of Technology, Tehran, Iran, in 1995 and 1997, respectively, and the Ph.D. degree in electrical engineering from The University of New South Wales, Sydney, Australia, in 2006.

She is currently a Postdoctoral Research Fellow with the Research School of Information Sciences and Engineering, The Australian National University, Canberra. From 1997 to 2002, she worked as a research engineer and then as a senior research engineer with Iran Communication Industries (ICI), Tehran, and with Deqx (Clarity Eq), Sydney. Her research interests include signal processing and channel modeling for wireless communication systems and also channel modeling and information theory of time varying fading channels. During her research career, she has visited various research institutes, including the Signal and Information Processing Laboratory, ETH, Zurich, Switzerland, in June 2006.

Dr. Sadeghi was a recipient of the Australian Postgraduate Award (APA) for the Ph.D. degree. In 2003 and 2005, she received two IEEE Region 10 Student Paper Awards for her research in the information theory of time-varying fading channels.

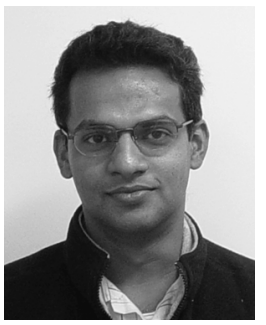

Thushara D. Abhayapala (M'94-S'97-M'00) received the B.E. degree (with honors) in interdisciplinary systems engineering in 1994, and the Ph.D. degree in telecommunications engineering from The Australian National University (ANU), Canberra, in 1999.

He is the Leader of the Wireless Signal Processing (WSP) Program at The National ICT Australia (NICTA). He is also an Associate Professor at ANU. From 1995 to 1997, he was a Research Engineer with the Arthur C. Clarke Center for Modern Technologies, Sri Lanka. Since December 1999, he has been a faculty member with the Research School of Information Sciences and Engineering, ANU. His research interests are in the areas of space-time signal processing for wireless communication systems, spatiotemporal channel modeling, array signal processing, and acoustic signal processing. He has supervised 17 research students and coauthored approximately 100 peer-reviewed papers.

Dr. Abhayapala is currently an Associate Editor for the EURASIP Journal on Wireless Communications and Networking.

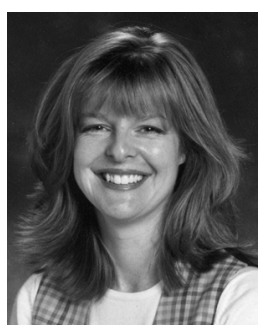

Haley M. Jones (M'01) received the Ph.D. degree in telecommunications engineering and wireless communications from the Australian National University, Canberra, in October 2002.

She is currently working as a lecturer with the Department of Engineering, Australian National University. Prior to the Ph.D. degree, she was working in industry and for a cooperative research center. Her research interests include wireless ad hoc networks, channel modeling, and channel characteristics in $a d$ hoc network routing protocols. 